\title{
Autophagy-dependent regulatory T cells are critical for the control of graft-versus-host disease
}

Laëtitia Le Texier, ${ }^{1}$ Katie E. Lineburg, ${ }^{1}$ Benjamin Cao, ${ }^{2,3}$ Cameron McDonald-Hyman, ${ }^{4}$ Lucie Leveque-El Mouttie, ${ }^{1}$ Jemma Nicholls, ${ }^{1}$ Michelle Melino, ${ }^{1}$ Blessy C. Nalkurthi, ${ }^{1}$ Kylie A. Alexander, ${ }^{1}$ Bianca Teal, ${ }^{1}$ Stephen J. Blake, ${ }^{1}$ Fernando Souza-Fonseca-Guimaraes, ${ }^{1}$ Christian R. Engwerda, ${ }^{1}$ Rachel D. Kuns, ${ }^{1}$ Steven W. Lane, ${ }^{1,5}$ Michele Teng, ${ }^{1}$ Charis Teh, ${ }^{7}$ Daniel Gray, ${ }^{6,7}$ Andrew D. Clouston, ${ }^{8}$ Susan K. Nilsson, ${ }^{2,3}$ Bruce R. Blazar, ${ }^{4}$ Geoffrey R. Hill, ${ }^{1,5}$ and Kelli P.A. MacDonald ${ }^{1}$

IImmunology Department, QIMR Berghofer Medical Research Institute, Brisbane, Queensland, Australia.

${ }^{2}$ Manufacturing, Commonwealth Scientific and Industrial Research Organization (CSIRO), Melbourne, Victoria, Australia. ${ }^{3}$ Australian Regenerative Medicine Institute, Monash University, Melbourne, Victoria, Australia. ${ }^{4}$ Pediatric Blood and Marrow Transplantation Program, University of Minnesota, Minneapolis, Minnesota, USA. ${ }^{5}$ Department of Bone Marrow Transplantation, Royal Brisbane Hospital, Brisbane, Queensland, Australia. ${ }^{6}$ Department of Medical Biology, University of Melbourne, Melbourne, Victoria, Australia. ${ }^{7}$ Molecular Genetics of Cancer Division and Immunology Division, The Walter and Eliza Hall Institute of Medical Research, Melbourne, Victoria, Australia. ${ }^{8}$ Envoi Specialist Pathologists, Brisbane, Queensland, Australia.

Regulatory $T$ cells (Tregs) play a crucial role in the maintenance of peripheral tolerance. Quantitative and/or qualitative defects in Tregs result in diseases such as autoimmunity, allergy, malignancy, and graft-versus-host disease (CVHD), a serious complication of allogeneic stem cell transplantation (SCT). We recently reported increased expression of autophagy-related genes (Atg) in association with enhanced survival of Tregs after SCT. Autophagy is a self-degradative process for cytosolic components that promotes cell homeostasis and survival. Here, we demonstrate that the disruption of autophagy within FoxP3 ${ }^{+}$Tregs (B6.Atg7//f/f-FoxP3cre $)$resulted in a profound loss of Tregs, particularly within the bone marrow (BM). This resulted in dysregulated effector $T$ cell activation and expansion, and the development of enterocolitis and scleroderma in aged mice. We show that the BM compartment is highly enriched in TICIT+ Tregs and that this subset is differentially depleted in the absence of autophagy. Moreover, following allogeneic SCT, recipients of grafts from B6.Atg $7^{1 / f-F}-$ FoxP3cre ${ }^{+}$donors exhibited reduced Treg reconstitution, exacerbated CVHD, and reduced survival compared with recipients of B6.WT-FoxP3cre ${ }^{+}$grafts. Collectively, these data indicate that autophagy-dependent Tregs are critical for the maintenance of tolerance after SCT and that the promotion of autophagy represents an attractive immune-restorative therapeutic strategy after allogeneic SCT.

Conflict of interest: The authors declare that no conflict of interest exists.

Submitted: February 3, 2016 Accepted: August 16, 2016 Published: September 22, 2016

Reference information: JCI Insight. 2016;1(15):e86850. doi:10.1172/jici.insight.86850.

\section{Introduction}

Allogeneic stem cell transplantation (SCT) remains an effective therapy for the majority of hematological malignancies. The curative property of SCT lies within the graft-versus-leukemia (GVL) effect, in which donor $\mathrm{T}$ and NK cells mediate clearance of residual tumor. The overall success of this procedure, however, is limited by graft-versus-host disease (GVHD), a serious complication with an associated high mortality rate. GVHD is a consequence of the priming of donor $\mathrm{T}$ cells against host antigens culminating in host target organ damage through both cytokine secretion and cytolysis.

FoxP3 $3^{+}$Tregs are an immunosuppressive $\mathrm{CD}^{+} \mathrm{T}$ cell population that function to constrain effector $\mathrm{T}$ cell responses, and they play an essential role in peripheral tolerance. The critical role of Tregs in the establishment and maintenance of tolerance after SCT is well documented (1). Indeed, Treg defects are commonly observed in the setting of clinical chronic GVHD (cGVHD), and recent clinical studies demonstrated the ability of low-dose IL-2 to expand Tregs and ameliorate cGVHD in a significant subset 
of patients (2-4). Multiple preclinical studies have highlighted the importance of Tregs in constraining GVHD, and the adoptive transfer of Tregs has been shown to attenuate both the acute (aGVHD) and cGVHD that manifest after SCT (5-10). While the adoptive transfer of Tregs after SCT has the therapeutic potential to promote tolerance, the low frequency of these cells, the time required to expand them in vitro to relevant numbers, and their instability after transfer represent limitations for the translation of Treg-based immunotherapy into the clinic. Furthermore, the processes that control Treg development, homeostasis, stability, and survival in vivo remain poorly defined. A better understanding of these processes is crucial to the establishment of logical therapeutic strategies to generate tolerance.

Granulocyte colony-stimulating factor (G-CSF) is commonly used to mobilize hematopoietic stem cells (HSCs) from the bone marrow (BM) into the peripheral blood (PB) to allow aphaeresis and collection as a stem cell source (G-PBSC) (11). G-CSF results in HSC mobilization in part through the disruption of CXCL12-CXCR4 interactions (12) that contribute to HSC homing and retention within the BM niche (13, 14). It is also clear that G-CSF imparts significant immunomodulatory effects on the graft that differentially impact aGVHD and cGVHD. Preclinical studies have demonstrated that on a per cell basis, T cells from G-CSF-mobilized donors have a reduced capacity to induce aGVHD compared with those from untreated donors $(15,16)$. In contrast, both preclinical and clinical studies demonstrate increased cGVHD, at least in part by the promotion of IL-17-dependent response cell differentiation $(11,17,18)$.

We have previously reported that stem cell mobilization with G-CSF protects against experimental GVHD, due, in part, to its effects on Tregs $(15,19)$. G-CSF mobilization increases Treg numbers in the blood of donors and in recipients after transplantation, suggesting a role for G-CSF in promoting Treg survival. Indeed, mRNA transcriptome analysis of Tregs after G-CSF demonstrates enhanced expression of autophagy-related genes (Atg), implicating a role for autophagy in this process (19). Autophagy is a self-degradative process for cytosolic components. It occurs after cellular stress induced by extra- and intracellular signals, including starvation, hypoxia, endoplasmic reticulum stress, and cytokines $(20,21)$. As such, autophagy plays an essential role in tissue and cell homeostasis and is considered an important survival mechanism. Recent studies have demonstrated the critical contribution of autophagy to the proliferation and survival of conventional T cells (Tcon cells) following their activation (22) and a role for autophagy in the maintenance of Tregs (23). Here, we confirm and extend this finding, demonstrating that autophagy is critical for the survival of $\mathrm{TIGIT}^{+}$Tregs that are enriched in the BM. We also confirmed that therapeutic doses of G-CSF mobilize Tregs into the periphery and induce autophagy. Moreover, Treg-intrinsic autophagy promotes their reconstitution following SCT and is required for the attenuation of GVHD. Collectively, these data indicate that autophagy-dependent Tregs are critical for optimal outcomes following SCT.

\section{Results}

Autophagy is an active process in Tregs and is required for their maintenance in the periphery. Microtubule-associated protein light chain 3 (LC3) is an autophagy-related protein that is incorporated into autophagosome membranes, and LC3 localization and quantification are commonly used to monitor autophagic activity (24). Using standard flow cytometry, we demonstrated increased LC3 expression in splenic CD $4^{+} \mathrm{CD} 25^{+}$ Tregs compared with $\mathrm{CD} 4^{+} \mathrm{CD} 25^{\text {neg }}$ Tcon cells from naive LC3-GFP mice (Figure 1A). Moreover, using LC3 staining and imaging flow cytometry, we confirmed an increased number of autophagosomes in Tregs (Figure 1B). Furthermore, short-term in vitro incubation with chloroquine (CQ), an inhibitor of autophagosome turnover, demonstrated enhanced accumulation of autophagosomes and thus autophagic flux in Tregs (Figure 1B). To assess the requirement for autophagy in Tregs in vivo, we used mice harboring a global deficiency in the autophagy-associated gene Atg5. Since global Atg5 deficiency is neonatal lethal, we generated Atg5 fetal liver chimera (FLC) mice, in which Atg5 deficiency is hematopoietic restricted. In the Atg $5^{-1-}$ FLC mice, the cellularity of thymus, spleen, and BM was similar to that in wild-type (WT) FLC mice. In contrast, whereas $\mathrm{CD}^{+} \mathrm{FoxP}^{+}$Treg frequencies and number were similar in the thymus, this population was significantly reduced in the spleen and BM of $A \operatorname{tg} 5^{-1-}$ FLC (Figure 1, C and D).

As autophagy-independent roles for ATG5 have been reported (25), we confirmed the requirement for autophagy in Tregs in a second model of hematopoietic autophagy deficiency. We generated BM chimeric

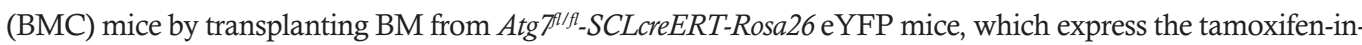
ducible Cre-ERT recombinase under the control of the stem cell enhancer of the stem cell leukemia ( $S c l$ ) locus (HSC-SCL-Cre-ERT), into congenic recipients. In these mice, HSCs could be rendered Atg7 deficient and tracked by way of an induced YFP reporter following tamoxifen administration. As observed in $\operatorname{Atg} 5^{-1-}$ FLC 

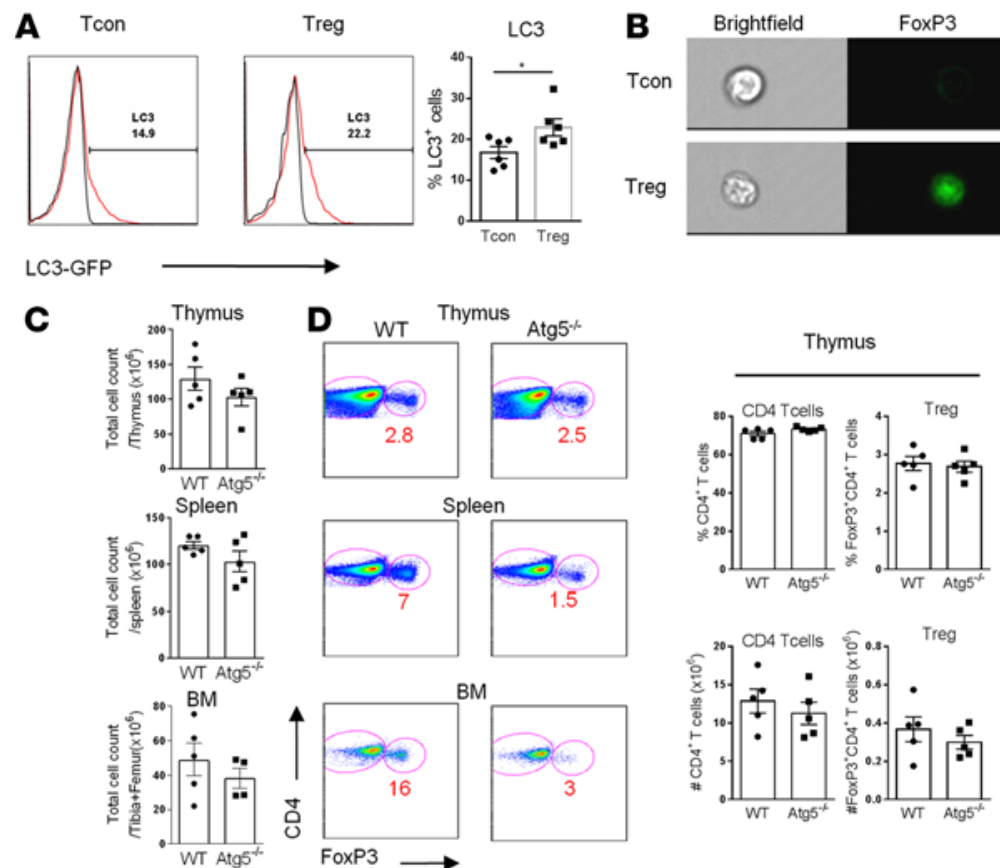

E

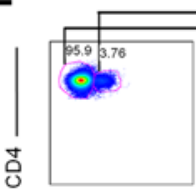

YFP
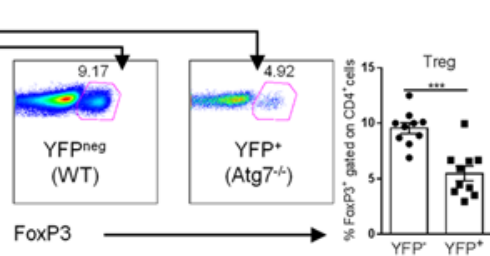

F
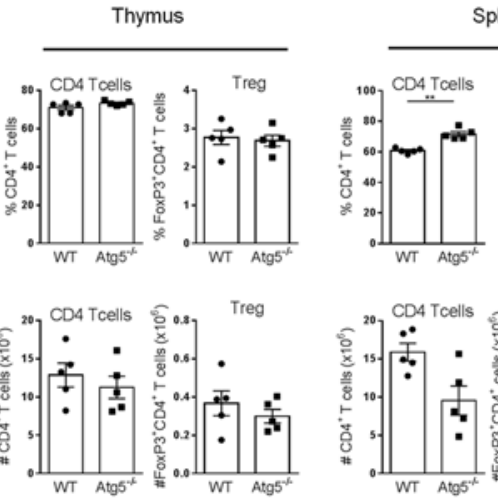

Spleen


Spleen
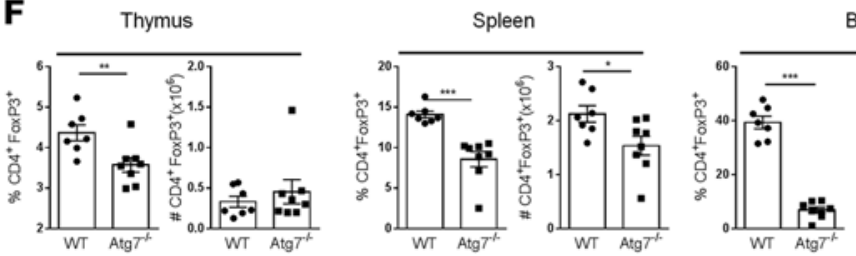

BM

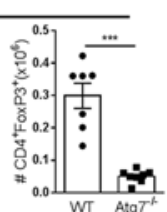

Figure 1. Autophagy is an active process in Tregs and required for their maintenance in the periphery. (A and B) Analysis of freshly isolated splenic CD4+CD25- Tcon and CD4+CD25+ Tregs from LC3-GFP mice. (A) Representative histograms of flow cytometry analysis for LC3 expression. Frequency of LC3 cells in CD4+CD25- Tcon cells and CD4+CD25+ Tregs (5 independent experiments; $n=6$ ). (B) Representative pictures of imaging flow cytometry (AMNIS) analysis of LC3 puncta formation in CD4 ${ }^{+} \mathrm{FoxP}^{\text {neg }}$ Tcon and CD4 ${ }^{+} \mathrm{FoxP3}^{+}$Treg populations isolated from FoxP3-GFP mice incubated with or without CQ. Mean of LC3 puncta/cell ( $n=6$ from 3 independent experiments). (C) Total thymus, spleen, and BM cell counts in WT ( $n=5)$ and Atg5 $5^{-1-}$ FLC mice $(n=5$ from 2 independent experiments). (D) Representative dot plot of flow cytometry analysis of FoxP3 ${ }^{+}$cells in CD4+CD8-CD3+CD45.2+CD45.1- $T$ cells. Frequency (\%) and absolute number (\#) of total CD4 ${ }^{+}$T cells and CD4+FoxP3 ${ }^{+}$Tregs in thymus, spleen, and BM of WT and Atg5 $5^{--}$FLC mice ( $n=5$ from 2 independent



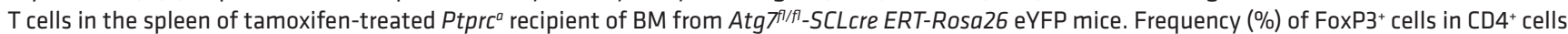
( $n=10$ from 2 independent experiments). (F) Frequency (\%) and absolute number (\#) of FoxP3 ${ }^{+}$T cells in CD4+CD8-CD3 ${ }^{+}$cells of thymus, spleen, and BM

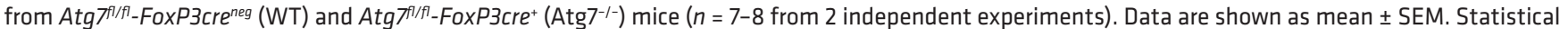
significance was determined using an unpaired 2-tailed Mann-Whitney $U$ test $\left({ }^{*} P<0.05 ;{ }^{* *} P<0.01 ;{ }^{* * *} P<0.001\right)$. Statistical analyses were performed using GraphPad Prism version 6.01 software. LC3, microtubule-associated protein light chain 3; Tcon, conventional T cells; CQ, chloroquine; Atg, autophagyrelated gene; FLC, fetal liver chimeras; YFP, yellow fluorescent protein.

mice, the frequency of Tregs in the $\mathrm{YFP}^{+} \mathrm{Atg} 7^{-/-} \mathrm{CD} 4^{+} \mathrm{T}$ cell compartment was significantly reduced compared with the frequency within $\mathrm{YFP}^{\mathrm{neg}} \mathrm{WT} \mathrm{CD} 4^{+} \mathrm{T}$ cells, confirming a functional role for autophagy in the maintenance of Tregs (Figure 1E). Diminished Treg numbers in the $\mathrm{Atg}^{7 / f-F}-F_{0} \times \mathrm{Pcre}^{+}$mice is likely a cumulative result of both cell death and Treg conversion, as Wei et al (23) reported that Treg-intrinsic autophagy contributes to both Treg survival and lineage stability. To establish a cell-intrinsic requirement for autophagy in Tregs, we generated $\mathrm{Atg}^{7 / f f}-\mathrm{FoxP}_{\mathrm{Cre}} \mathrm{C}^{+}$mice, in which ATG7 is disabled specifically in FoxP3 ${ }^{+}$Tregs. Similar to $\mathrm{Atg}^{-1-}$ FLC mice, a Treg deficiency in the periphery was observed, while thymic Tregs remained intact. Notably, in Atg $7^{7 / f}$-FoxP $3 \mathrm{cre}^{+}$mice, there was a more modest reduction in Treg numbers in the spleen compared with the BM ( $30 \%$ vs. $85 \%$, respectively) (Figure $1 \mathrm{~F})$. As thymic Treg generation appeared intact, we next investigated the requirement of autophagy for in vitro differentiation of naive $\mathrm{CD} 4^{+} \mathrm{T}$ cells into induced Tregs (iTregs). We demonstrated the equivalent capacity of $\mathrm{CD} 4^{+} \mathrm{CD} 25^{\text {neg }} \mathrm{T}$ (Tcon) cells from either $\operatorname{Atg} 5^{-1-} \mathrm{FLC}$ or WT FLC mice to differentiate into iTregs, without significant differences in their frequency, absolute number, or FoxP3 expression (Supplemental Figure 1A; supplemental material available online with this article; doi:10.1172/ jci.insight.86850DS1). These findings were confirmed by generating iTregs using autophagy-replete Tcon sort 
A

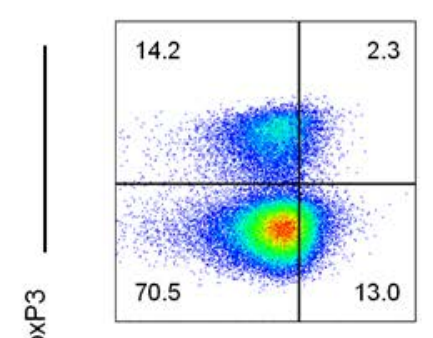

MCL-1

B

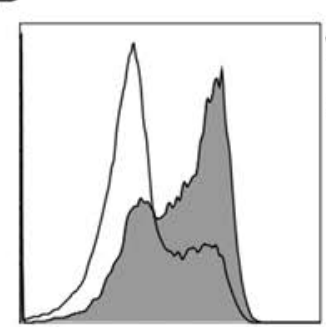

$\operatorname{Atg} 7^{*}$
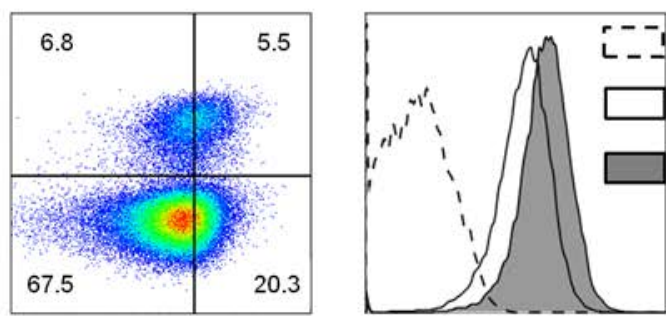

MCL-1



Ki67

C

WT
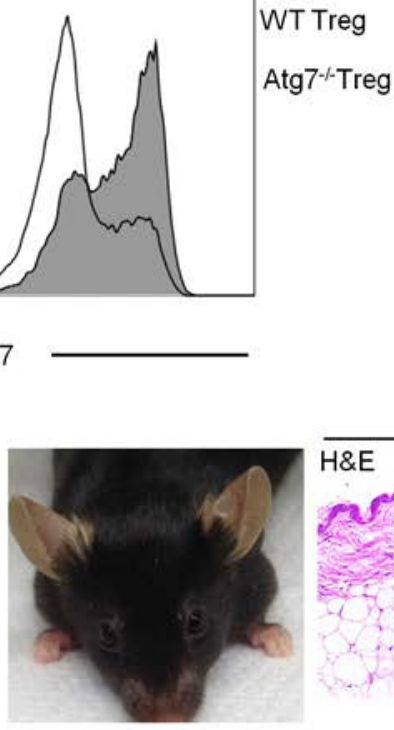

Ki67

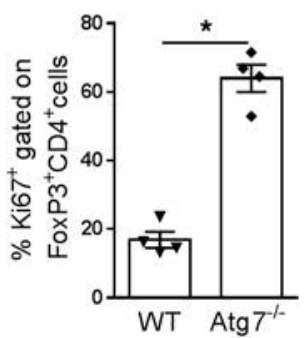

SI Liver
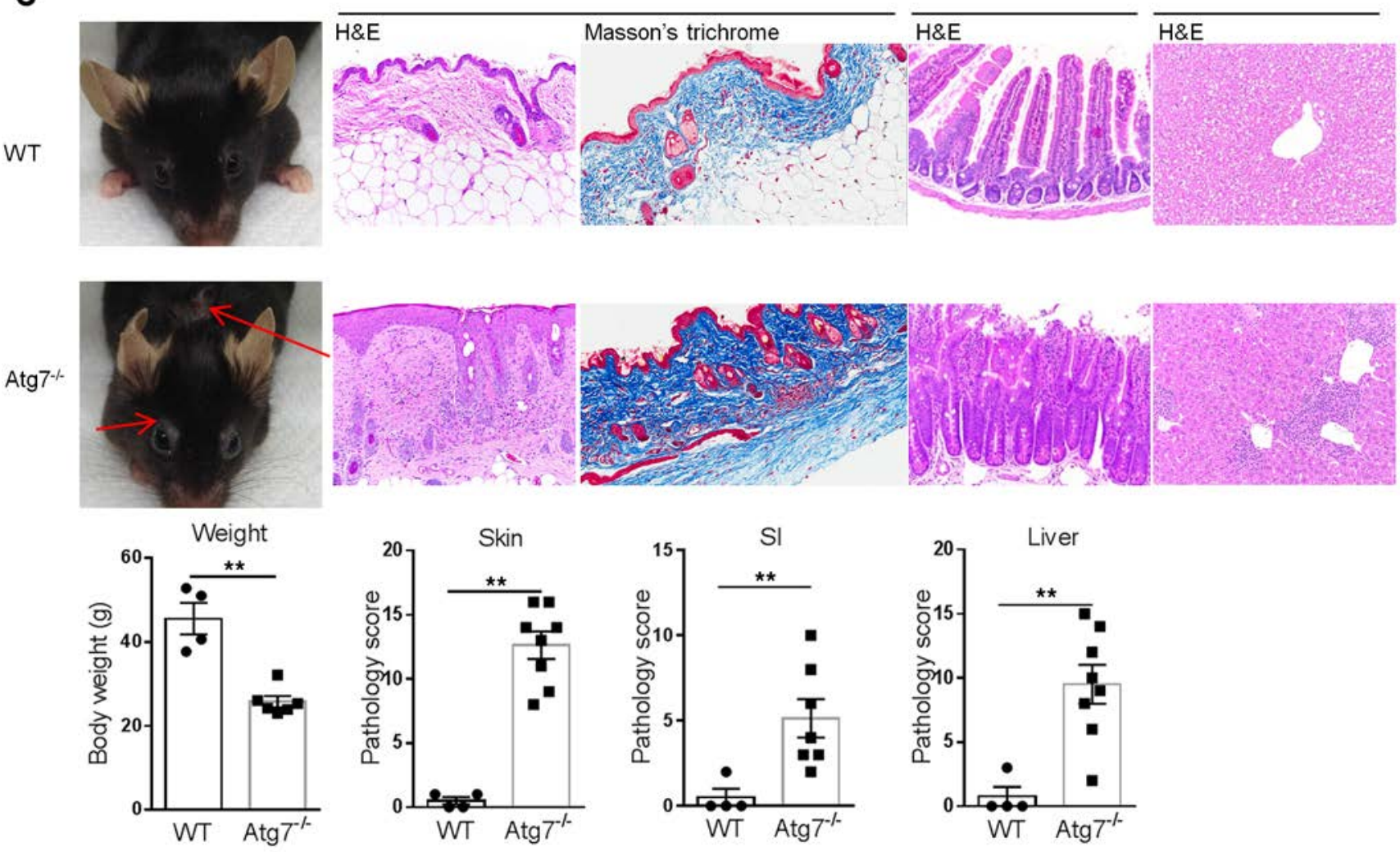

Figure 2. Treg-intrinsic autophagy deficiency results in immune pathology in aged mice. (A and B) Flow cytometry analysis of WT-FoxP3cre ${ }^{+}(\mathrm{WT})$ and Atg $7^{f / f-1}-F_{0 x P 3 C r e^{+}}\left(\mathrm{Atg}^{-I^{-}}\right)$mice ( $n=4$ from 2 independent experiments). (A) Representative dot plot of MCL-1 and FoxP3 ${ }^{+}$expression in $\mathrm{CD}^{+} \mathrm{CD}^{-} \mathrm{CDB}^{+}$ splenic T cells. Representative histogram and geometric mean of MCL-1 expression in FoxP3 ${ }^{+} \mathrm{CD} 4^{+} \mathrm{CD} 88^{-} \mathrm{CD} 3^{+}$splenic T cells. (B) Representative histogram

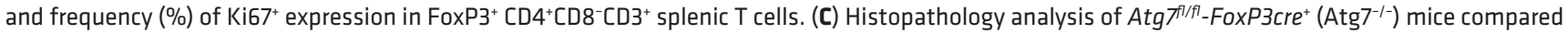
with WT-FoxP3cre+ (WT) mice. Representative pictures of aged mice (32-44 weeks). Representative images of H\&E and Masson's trichrome staining of skin samples. Representative images of H\&E staining and pathology score of skin, small intestine (SI), and liver samples $\left(n=4\right.$, WT; $n=7-8$, Atg7 $\left.7^{-1-}\right)$. Original magnification, $\times 200$. Body weight of mice $(30-37$ weeks) $(n=4-6)$. Data are shown as mean $\pm \mathrm{SEM}$. Statistical significance was determined using an unpaired 2-tailed Mann-Whitney $U$ test $\left({ }^{*} P<0.05\right.$, $\left.{ }^{*} P<0.01\right)$. Statistical analyses were performed using GraphPad Prism version 6.01 software. Atg, autophagy-related gene; MCL-1, myeloid cell leukemia 1. 
A

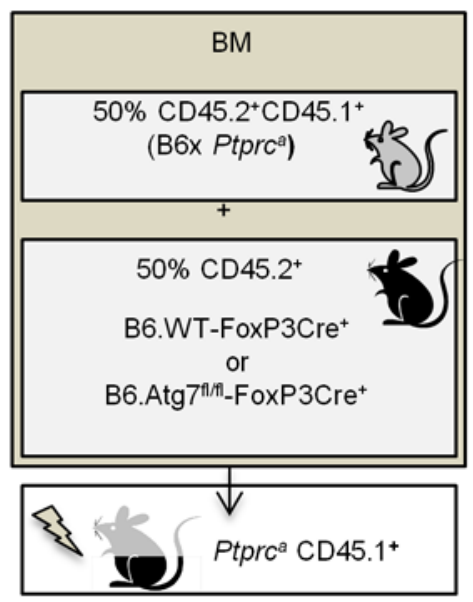

C


\# TIGIT ${ }^{+}$
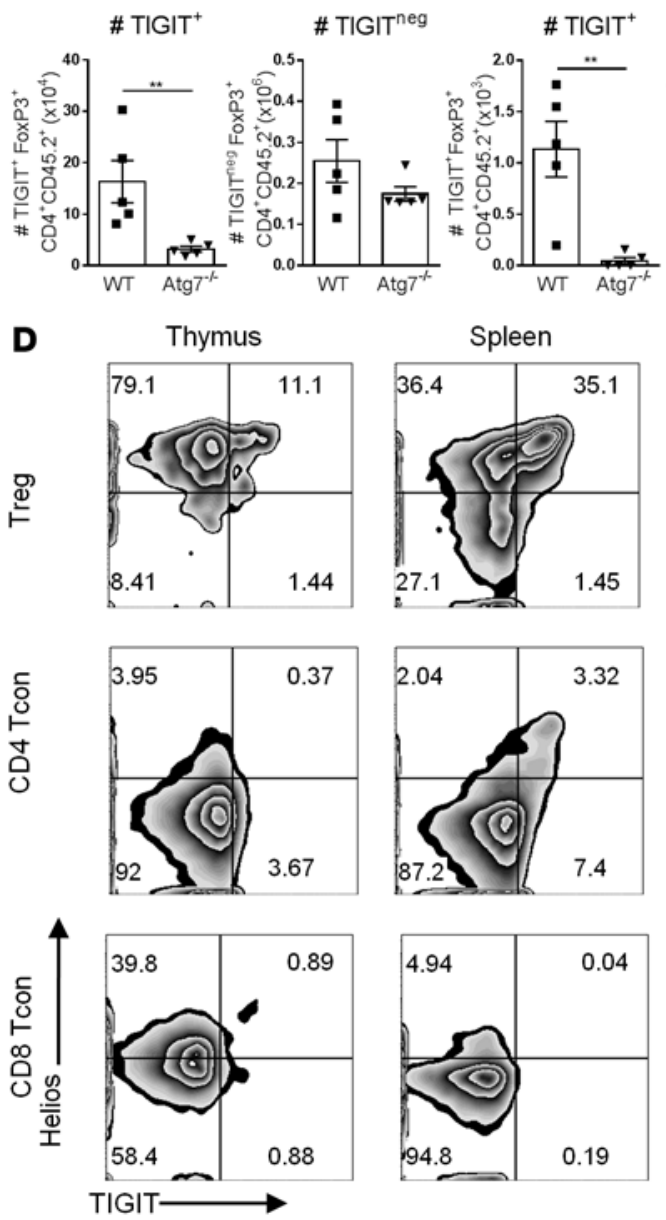
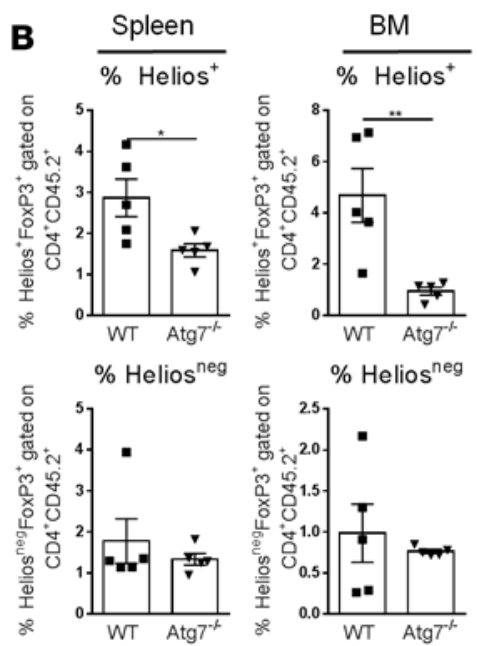

BM

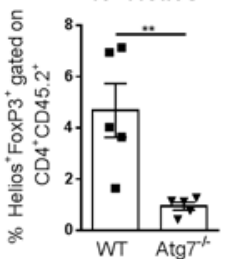

$\%$ Helios $^{\text {neg }}$
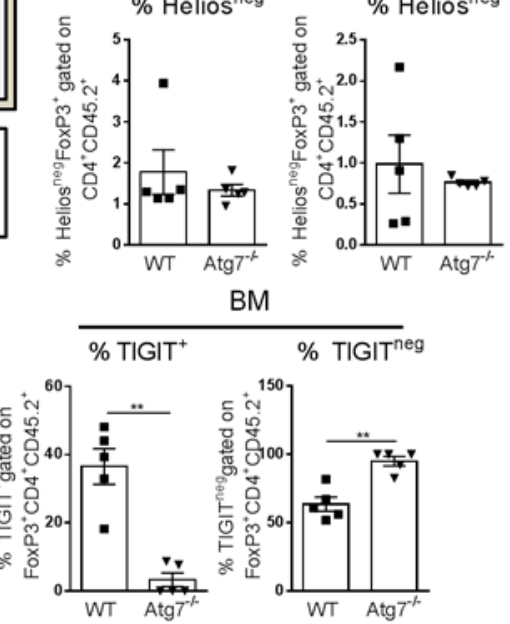

\# TIGIT ${ }^{\text {neg }}$


Figure 3. Autophagy is required to maintain the CD4 $^{+}$FoxP3 ${ }^{+}$Helios ${ }^{+}$TIGIT+ $^{+}$Treg subset. (A-C) Cytometry analysis of Tregs from Atg $^{-1-}$ and WT mixed chimera generated by injecting $B M$ from Atg $7^{f / / f}-F_{0 x P 3} \mathrm{cre}^{+}$ (CD45.2 (Atg7 $^{-I^{-}}$) or WT-FoxP3Cre (CD45.2 $^{+}$(WT) mice with an equal number of $B M$ cells from congenic (CD45.1+CD45.2+) mice into irradiated syngeneic Ptprc ${ }^{a}$ mice $\left(C D 45.1^{+}\right)(n=5)$. (A) Outline of mixed chimera mouse transplant strategy. (B) Frequency (\%) of Helios ${ }^{+}$ and Helios ${ }^{\text {neg }}$ and (C) frequency (\%) and absolute number (\#) of TICIT+ and TIGIT ${ }^{\text {neg }}$ Tregs gated on Fox$\mathrm{P3}^{+}{ }^{+}{ }^{-} 4^{+} \mathrm{CD}^{-} \mathrm{CDO}^{+} \mathrm{CD} 45.2^{+}$cells in spleen and BM. (D) Representative zebra plot of flow cytometry analysis and frequency (\%) of Helios and TIGIT expression on FoxP3 ${ }^{+} \mathrm{CD} 4^{+} \mathrm{CD}^{-} \mathrm{CD3}^{+}$(Tregs), FoxP3 ${ }^{\text {nes }} \mathrm{CD}^{+} \mathrm{CD}^{+} \mathrm{CD} 3^{+}$ (CD4 Tcon), and FoxP3 ${ }^{\text {neg }} \mathrm{CD} 8+{ }^{+} \mathrm{CD} 4-\mathrm{CD3}^{+}$(CD8 Tcon) cells analyzed in thymus, spleen, and BM of FoxP3-GFP mice ( $n=4-8$ from 2 independent experiments). Data are shown as mean \pm SEM. Statistical significance was determined using an unpaired 2-tailed Mann-Whitney $U$ test $\left({ }^{*} P<0.05 ;{ }^{* *} P<0.01 ;{ }^{* * *} P<0.001\right)$. Statistical analyses were performed using GraphPad Prism version 6.01 software. Atg, autophagy-related gene; TICIT,

T cell immunoreceptor with Ig and ITIM domains; Tcon, conventional T cells.

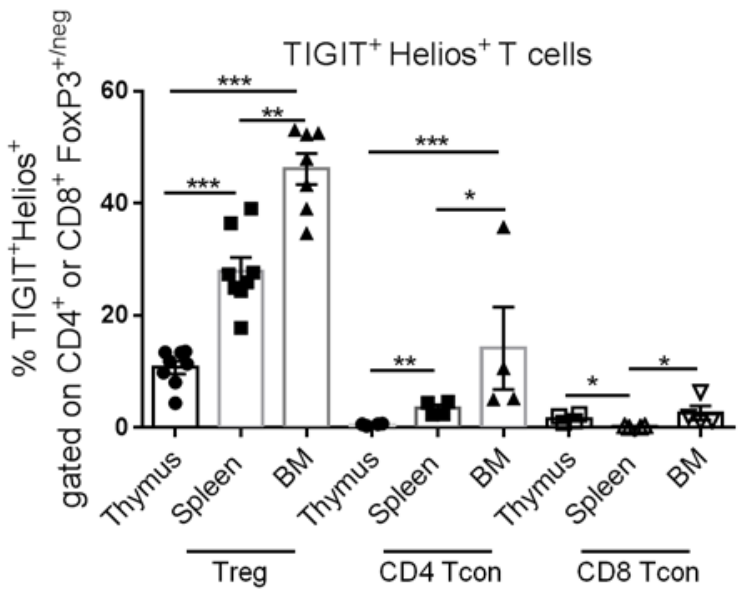


A
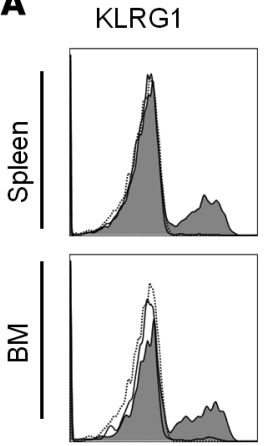

B


CD62L
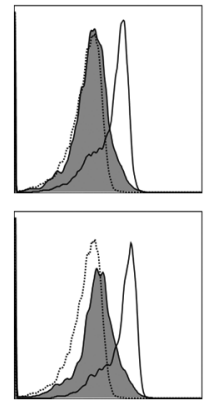

CD73

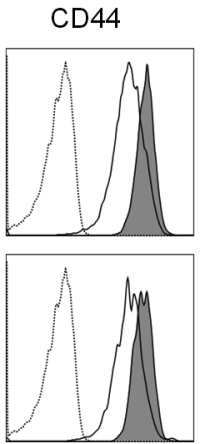

GITR
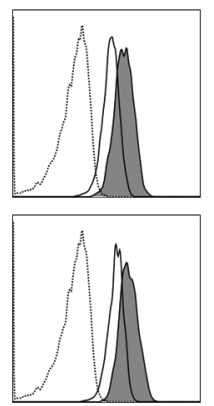

CD69
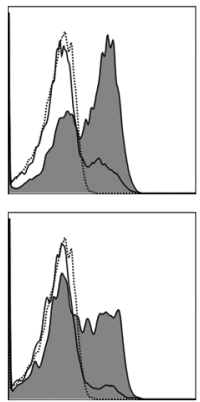

ICOS
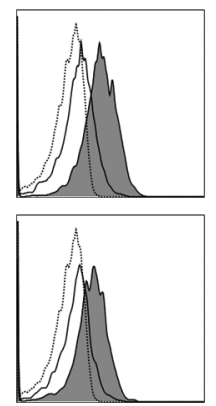


CTLA4


i-n Isotype

$\square$ TIGITneg

$\square \mathrm{TIGIT}^{+}$
C



BCL-2

$\mathrm{BM}$



I- $\mathbf{I}$ I Isotype $\square$ TIGIT neg $\square$ TIGIT+ $^{+}$

D



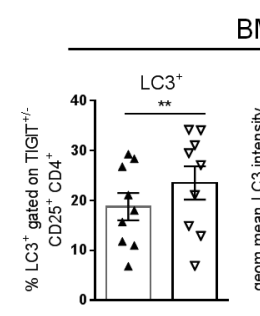

BM
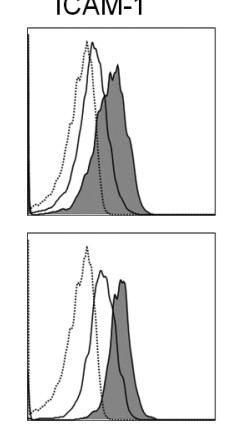

IL-10
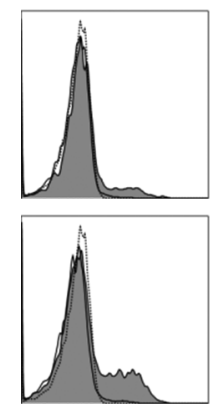

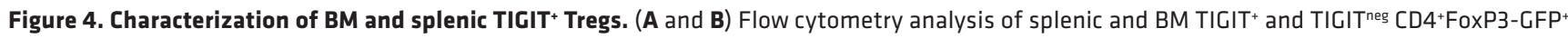
or FoxP3-RFP+ Tregs from FoxP3-GFP or IL-10-GFP-FoxP3-RFP naive mice. Expression of KLRG1, CD62L, CD44, CD69, CD25, PD-1, CD73, GITR, ICOS, CTLA4, ICAM-1, and IL-10. The isotype control represents staining of pooled BM and spleen cells. Representative histograms of phenotype analysis ( $n$ = 3 from 3 independent experiments). (C) Representative histograms of BCL-2 expression in splenic and BM TIGIT+ and TIGIT neg $\mathrm{CD}^{+}{ }^{+}$FoxP3 ${ }^{+}$cells from C57BL/6 naive mice ( $n=3$ from 1 experiment). (D) Frequency (\%) and intensity (geometric mean) of LC3 expression in splenic and BM TIGIT+ and TIGITes CD4 ${ }^{+} C D 25^{+}$Tregs from LC3-GFP mice ( $n=9$ from 3 independent experiments). (E) ATG7 transcripts quantification by RT-qPCR in sorted TIGIT ${ }^{+}$and TIGIT $^{\text {neg }}$ CD4 ${ }^{+}$FoxP3-GFP+ splenic Tregs from FoxP3-GFP naive mice ( $n=6$ from 3 independent experiments). Data are shown as mean \pm SEM. Statistical significance was determined using a Wilcoxon matched-paired test $\left({ }^{*} P<0.05 ;{ }^{* *} P<0.01\right)$. Statistical analyses were performed using GraphPad Prism version 6.01 software. TIGIT, T cell immunoreceptor with Ig and ITIM domains; RFP, red fluorescent protein; KLRG1, coinhibitory receptor killer cell lectin-like receptor G1; PD-1, programmed cell death protein 1; GITR, glucocorticoid-induced TNFR-related protein; ICOS, inducible T cell costimulator; CTLA4, cytotoxic T lymphocyte-associated protein 4; ICAM-1, intercellular adhesion molecule 1; BCL-2, B cell lymphoma 2; LC3, microtubule-associated protein light chain 3; Atg, autophagy-related gene.

purified from $A \operatorname{tg} 7^{1 / f-F}$-FoxP3cre ${ }^{+}$and $W T$-FoxP3cre ${ }^{+}$mice (Supplemental Figure 1B). These data demonstrate that autophagy is dispensable for the generation of thymic derived Tregs and peripheral Treg differentiation, but is required for maintenance of Tregs in the periphery, particularly within the BM.

Treg homeostasis is a tightly controlled process regulated by IL-2 signals and the intrinsic expression of the antiapoptotic protein MCL-1 (26). In this regard, following even partial Treg depletion in vivo, residual Tregs undergo niche-filling behavior to rapidly reconstitute Treg numbers. We reasoned that loss of Tregs, which occurs in the setting of intrinsic Treg autophagy deficiency, would elicit niche-filling behavior in residual Tregs. We confirmed increased Treg proliferation (Ki67) and MCL-1 expression in Tregs from $\mathrm{Atg}^{1 / f-}$-FoxP3Cre ${ }^{+}$mice (Figure 2, A and B), demonstrating intact deficit-sensing activity; however, this was not sufficient to overcome the Treg deficit in these animals. These data confirm a disruption of homeostasis in the setting of Treg-intrinsic autophagy deficiency. 
A



B



Spleen
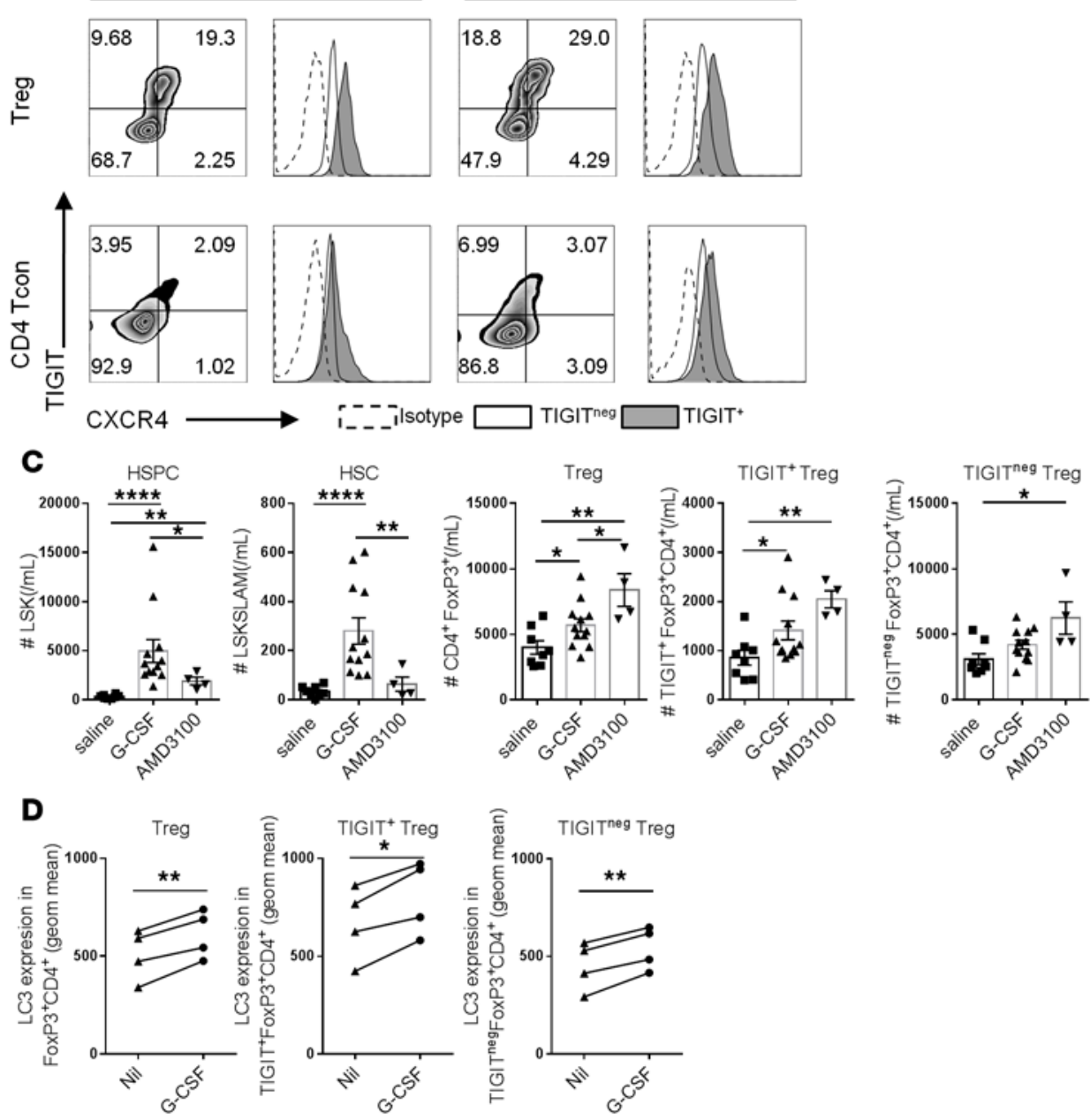

TIGIT+ Treg $\quad$ TIGIT $^{\text {neg }}$ Treg


Treg -intrinsic autophagy deficiency results in immune pathology in aged mice. Although a Treg deficiency in

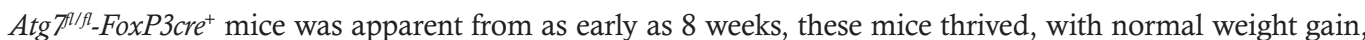
and showed only sporadic and mild histological signs of immune pathology until approximately 4 months of age (data not shown). However, from 16 weeks onward, Atg $7^{f / f}-F o x P 3 c r e^{+}$mice spontaneously developed multi-organ inflammation which was most severe in the skin and gastrointestinal (GI) tract. The severity of disease increased with age, and from 32 weeks onward, Atg $7^{\prime / f-}-F o x P 3 c r e^{+}$mice exhibited weight loss and macroscopic evidence of skin disease, including alopecia and lesions (Figure 2C). Histological analysis of tissue at this point revealed severe scleroderma characterized by marked collagen deposition, and extensive inflammation and architectural disruption in the GI tract and liver (Figure 2C). Examination of the 
immune status of mice (at 13-26 weeks of age), prior to the development of overt pathology, demonstrated significantly increased expansion, activation, and proliferation of $\mathrm{CD}^{+} \mathrm{FoxP}^{\text {neg }}$ Tcon cells together with an increased proportion of effector memory cytokine-producing $\mathrm{CD}^{+}$and $\mathrm{CD} 8^{+} \mathrm{T}$ cells (Supplemental Figure 2, A-E, and data not shown). The fact that $\mathrm{T}$ cell activation occurred despite only a $30 \%$ reduction in Tregs in the periphery of $A \operatorname{tg} 7^{\prime / f l}-F o x P 3 c r e^{+}$mice led us to investigate the impact of autophagy on Treg function. Surprisingly, compared with their WT controls, freshly isolated Atg $7^{f l f l}-F o x P 3 c r e^{+}$Tregs and $A \operatorname{tg} 5^{-1-}$ CD $4^{+}$ FoxP3 ${ }^{+}$iTregs were equally capable of suppressing effector T cells in vitro (Supplemental Figure 3, A and B).

Autophagy is required to maintain the $\mathrm{CD}^{+} \mathrm{FOXP}^{+} \mathrm{Helios}^{+} \mathrm{TIGIT}^{+}$Treg subset. As we observed that the decrease of Tregs in $A \operatorname{tg} 7^{f / f}-F_{-} x P 3 c r e^{+}$mice was neither complete nor equivalent across organs, we investigated whether autophagy was specifically required within a specific Treg subset. To avoid the potential influence of the inflammatory environment present in $A \operatorname{tg} 7^{f / f l}-F o x P 3 c r e^{+}$autoimmune-prone mice, we generated mixed $\mathrm{BMC}$ mice by reconstituting congenic $\left(\mathrm{CD} 45.1^{+}\right)$recipients with equal amounts of $\mathrm{BM}$ from WT-FoxP3cre $\left(\mathrm{CD} 45.2^{+}\right)$or Atg $7^{\text {flfl-}}-\mathrm{FoxP}_{\mathrm{cre}}{ }^{+}\left(\mathrm{CD} 45.2^{+}\right)$mice and CD 45.1 $1^{+} \mathrm{CD} 45.2^{+}$mice (Figure 3A). Of note, whereas engraftment of total CD45.2 $2^{+}$TT-FoxP3cre or Atg $7^{f / f}-F o x P 3 c r e$ cells was equivalent to WT $\left(\mathrm{CD} 45.1^{+} \mathrm{CD} 45.2^{+}\right)$cells in the mixed BMC mice (Supplemental Figure 4A), within the Treg compartment, both WT.FoxP3cre or Atg $7^{f / f l}-F o x P 3 c r e^{+}$Tregs were significantly reduced numerically compared with WT Tregs (Supplemental Figure 4, B and C). A recent study has demonstrated that the presence of the FoxP3cre allele significantly impacts WT Tregs, rendering them hypomorphic, with an associated decrease in FoxP3 expression (27). Thus, as the expression of FoxP3-associated cre in Tregs in either WT or Atg $7^{\text {flfl }}$-FoxP $3 \mathrm{cre}^{+}$ mice impacted the reconstitution fitness and potentially the phenotype of these cells, we compared the number and phenotype of WT-FoxP $3 \mathrm{cre}^{+}$and $A \operatorname{tg} 7^{\text {Ilfl}}-\mathrm{FoxP} 3 \mathrm{cre} \mathrm{C}^{+}$Tregs, avoiding comparison with the CD 45.1 $1^{+} \mathrm{CD} 45.2^{+}$WT (cre ${ }^{\text {neg }}$ ) Tregs. We first assessed the expression of Helios, the ikaros family transcription factor, which has been used to distinguish thymus-derived Tregs (Helios ${ }^{+}$Tregs) from Tregs induced in the periphery (Helios ${ }^{\text {neg }}$ Tregs) (28). However, more recently, Helios expression has been linked to activated Tregs (29) rather than thymic-derived Tregs. The analysis of mixed BMC shows a specific defect of Helios Tregs in the autophagy-deficient Treg population (Figure 3B). Furthermore, expression of the coinhibitory molecule TIGIT identifies a subset of activated Tregs implicated in the suppression of autoimmunity ( 30 , 31). Thus, we characterized this molecule within Tregs in these chimeric mice and identified the TIGIT $^{+}$ Treg population as strikingly decreased in spleen and BM, while the TIGIT ${ }^{\text {neg }}$ Treg population was relatively spared (Figure 3C). Of note, in the absence of autophagy, Treg deficiency was most striking in the BM, where Atg7 deficiency resulted in a 25 -fold decrease in the absolute number of TIGIT $^{+}$Tregs, compared with a 5 -fold decrease in the spleen (Figure 3C).

We noted a significant enrichment of TIGIT $^{+}$Tregs (which were all Helios ${ }^{+}$) within the BM of WT naive mice. Additionally, TIGIT ${ }^{+} \mathrm{CD}^{+}$, but not $\mathrm{CD}^{+}$, Tcon cells exhibited a similar profile of enrichment across the lymphoid organs (BM > spleen $>$ thymus), although the proportion of $\mathrm{TIGIT}^{+} \mathrm{CD}^{+}$ Tcon cells was significantly lower than that of TIGIT ${ }^{+}$Tregs (Figure 3D). In order to characterize the phenotype of $\mathrm{TIGIT}^{+}$and TIGIT $^{\text {neg }}$ Treg subsets in the spleen and BM compartment, we analyzed the expression of maturation, activation (KLRG1, CD62L, CD44, CD69, CD25), and functional (PD-1, CD73, GITR, ICOS, CTLA4, ICAM-1, IL-10) markers in FoxP3-GFP naive mice. In line with previous analyses of TIGIT $^{+}$Tregs (30), we observed a more activated, memory, and functional phenotype characterized by low CD62L and high CD44, CD69, KLRG1, PD-1, CD73, GITR, ICOS, CTLA4, ICAM-1, and IL-10 expression irrespective of their tissue localization (Figure 4, A and B). Notably, although TIGIT ${ }^{+}$Tregs $^{-}$ have previously been reported to express elevated levels of CD25 (30), we observed reduced cell surface CD25 expression on TIGIT $^{+}$Tregs compared with their TIGIT ${ }^{\text {neg }}$ counterparts. This discrepancy may reflect the different transgenic FoxP3 reporter lines used in these studies, as the introduction of the reporter modulates function in some strains $(27,32)$. We therefore confirmed the reduced expression of CD25 on TIGIT $^{+}$Tregs in nontransgenic WT C57BL/6 mice (Supplemental Figure 5). In view of their differential survival characteristics, we next analyzed BCL-2 expression, which was reduced in $\mathrm{TIGIT}^{+}$Tregs (Figure 4C). Moreover, the analysis of the LC3-GFP expression in both Treg subsets and quantification of ATG7 mRNA suggest higher basal levels of autophagy in TIGIT ${ }^{+}$Tregs (Figure 4, D and E), indicative of the utilization of different survival pathways.

G-CSF mobilizes Tregs from the BM and induces autophagy. In addition to mobilizing stems cells from the BM into the periphery, G-CSF administration mobilizes Tregs $(33,34)$. Indeed, the administration of G-CSF for 6 days resulted in a significant reduction in both TIGIT $^{+}$and TIGIT $^{\text {neg }}$ Tregs in the BM and an 
A

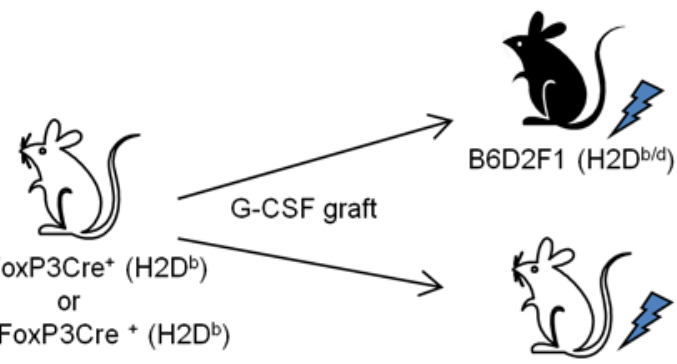

$\operatorname{Ptprc}^{\mathrm{a}}\left(\mathrm{H}_{2} \mathrm{D}^{\mathrm{b}}\right)$
B

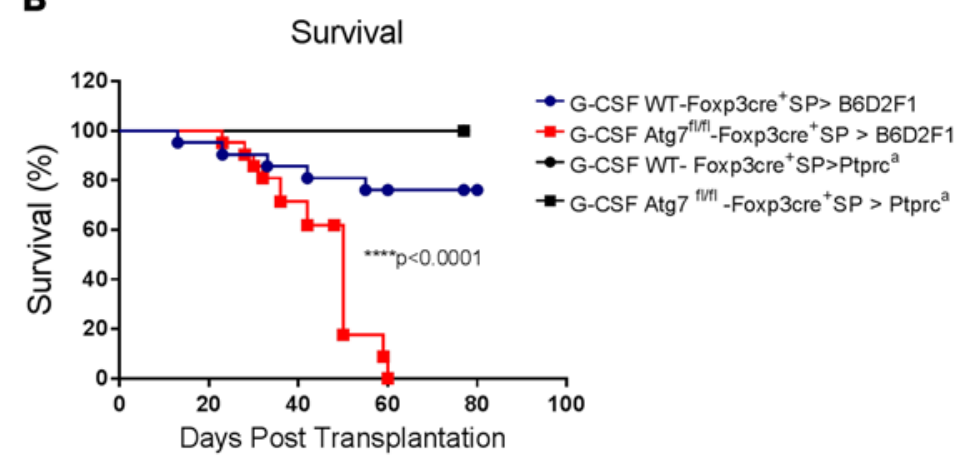

C



Liver

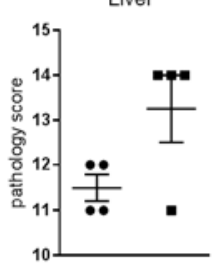

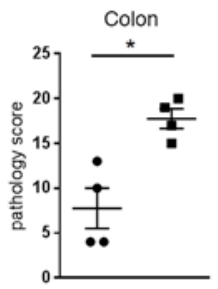

Skin



- WT-FoxP3cre ${ }^{+}$

- $A \operatorname{tg} 7^{\mathrm{ntr}}-$ FoxP3cre
D $\quad$\begin{tabular}{c} 
Tcon \\
\cline { 2 - 3 }
\end{tabular}
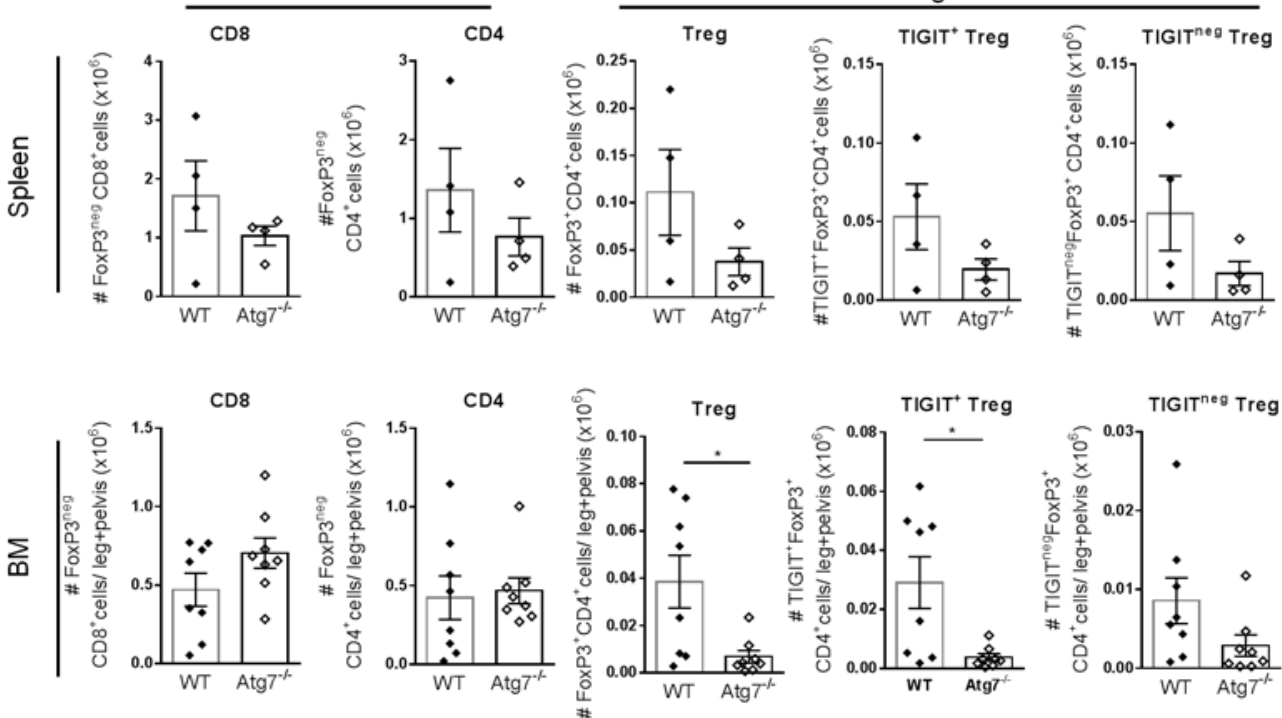

Figure 6. Autophagy-dependent BM Tregs are required to control GVHD after G-CSF-mobilized SCT. (A-D) Irradiated syngeneic $P$ tprc ${ }^{a}$ mice (CD45.1 $1^{+}, \mathrm{H}_{2} \mathrm{D}^{\mathrm{b}}$ )


WT-FoxP3cre $\left(C D 45.2^{+}, H 2 D^{b}\right)(W T)$ mice $(n=5)$. (A) Outline of SCT strategy. (B) Survival curve (\%) of allogeneic and syngeneic recipients after transplantation. Statistical significance was determined using log-rank (Mantel-Cox) test (**** $P<0.001)$. (C) Pathology score from histopathology analysis of small intestine, colon, liver, and skin of allogeneic recipients and representative images of H\&E staining of colon samples ( $n=4$ from 1 experiment). Original

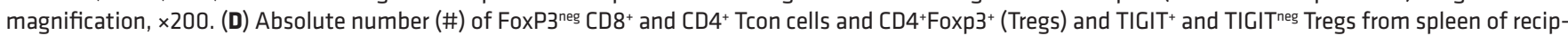
ients (representative of 2 independent experiments with $n=4$ in each experiments) and BM ( $n=8$ from 2 independent experiments) of allogeneic recipient mice 42 days after transplant. Data are shown as mean \pm SEM. Statistical significance was determined using an unpaired 2-tailed Mann-Whitney $U$ test $\left({ }^{*} P<0.05\right)$. Statistical analyses were performed using GraphPad Prism version 6.01 software. GVHD, graft versus host disease; G-CSF, granulocyte-colony stimulating factor; SCT, stem cell transplantation; Atg, autophagy-related gene; SP, spleen; TIGIT, T cell immunoreceptor with Ig and ITIM domains. 
A



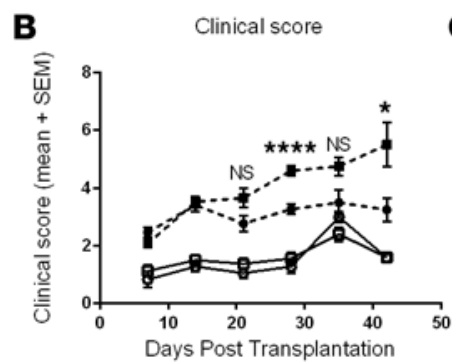

C Survival



D

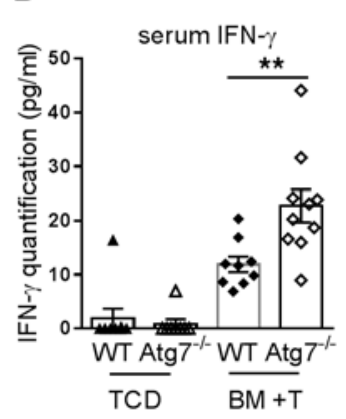

E
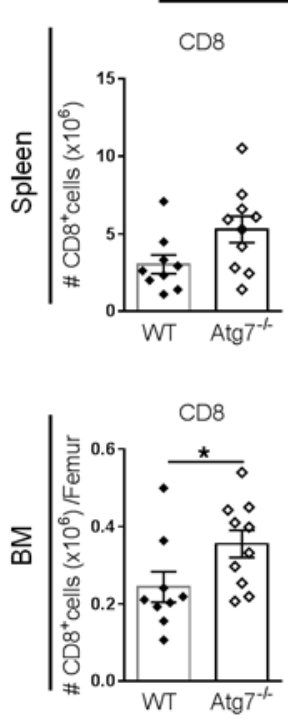

Tcon


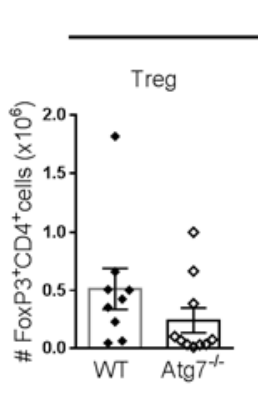

Treg
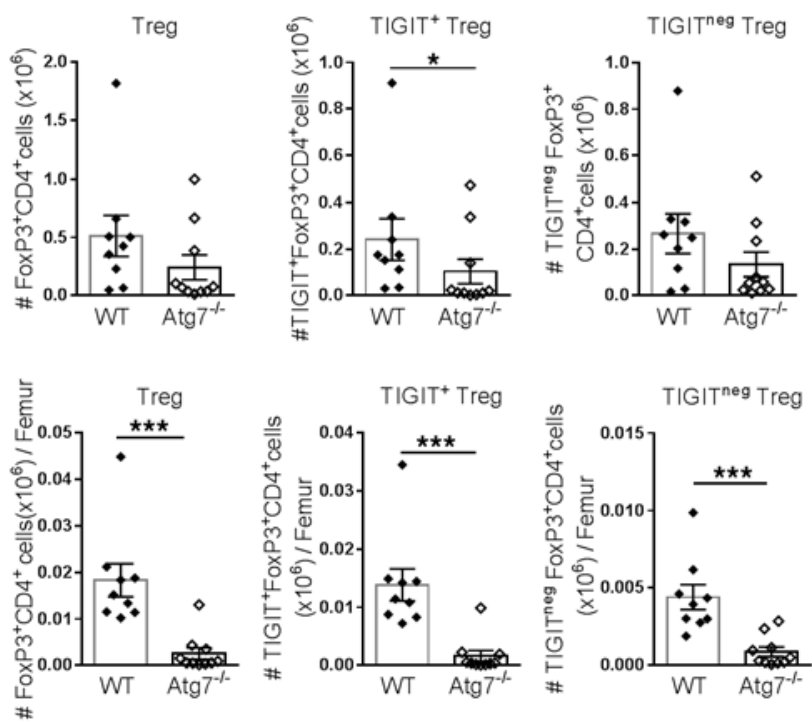

Figure 7. Autophagy-dependent BM Tregs are required to control CVHD and survival after BMT. (A-E) Irradiated allogeneic B6D2F1 (CD45.2+ $\left.\mathrm{H} 2 \mathrm{D}^{\mathrm{b} / \mathrm{d}}\right)$ recipient mice were transplanted with BM graft alone (T cell-depleted [TCD] BM) or BM + T cell graft $(B M+T)$ composed of sorted Tregs (YFP') + Tcon (YFPnes) cells from either Atg fl/fl-FoxP3cre $^{+}$(Atg7-/-) or WT-FoxP3cre mice (WT). Analysis at day 28 after transplantation (BM + T) or day 28 to 41 (BM TCD). (A) Outline of SCT strategy. (B) GVHD clinical scores of recipients after transplantation ( $\left.{ }^{* * *} P<0.0001\right)(n=6-16$ from 2 independent experiments). (C) Survival curve (\%) of recipient mice after transplantation. Statistical significance was determined using log-rank (Mantel-Cox) test $\left({ }^{*} P<0.05\right)$ ( $n=6$ from 1 experiment). (D) IFN- $\gamma$ quantification in sera ( $\left.{ }^{* *} P<0.01\right)\left(n=8-10\right.$ from 2 independent experiments). (E) Absolute number (\#) of CD8 ${ }^{+} C D 3^{+}$Tcon cells (CD8), FoxP3 ${ }^{\text {nes }} C D 44^{+} C D 3^{+}$Tcon cells (CD4), CD4+Foxp3 ${ }^{+}$Tregs (Tregs), and TIGIT+ and TIGITneg Tregs from spleen and BM ( $n=8-10$ from 2 independent experiments) of recipient mice in the BM + T setting. Data are shown as mean $\pm \mathrm{SEM}$. Statistical significance was determined using an unpaired 2-tailed Mann-Whitney $U$ test $\left({ }^{*} P<0.05 ;{ }^{* * *} P<0.001\right.$ ). Statistical analyses were performed using GraphPad Prism version 6.01 software. GVHD, graft versus host disease; BMT, bone marrow transplantation; YFP, yellow fluorescent protein; Tcon, conventional T cells; Atg, autophagy-related gene; TCD, T cell depleted; TIGIT, T cell immunoreceptor with Ig and ITIM domains.

enrichment of both TIGIT $^{+}$and TIGIT ${ }^{\text {neg }}$ Tregs (although only TIGIT $^{+}$reached significance) in the spleen (Figure 5A). In line with previous reports of elevated CXCR4 expression on activated Tregs (33), TIGIT $^{+}$ Tregs from both the spleen and BM expressed higher levels of CXCR4 compared with TIGIT ${ }^{\text {neg }}$ Tregs. Again, in parallel, elevated levels of CXCR4 were also associated with the $\mathrm{TIGIT}^{+} \mathrm{CD} 4^{+}$Tcon cells (Figure 5B). Thus, through their enhanced CXCR4 expression, TIGIT $^{+}$Tregs may preferentially home to and be retained in the $\mathrm{BM}$, explaining their enrichment at this site. As the disruption of the CXCR4/SDF-1 axis is the primary mechanism by which G-CSF administration releases hematopoietic stem cells from the BM, we next asked whether targeted disruption of this axis with the CXCR4 antagonist AMD3100 would also lead to Treg mobilization. One hour after administration of AMD3100, we observed a small but significant increase in both HSCs and progenitor (HSPC) populations within the PB, and that AMD3100 HSC mobilization was less efficient than G-CSF administration (administered twice a day for 4 days). Moreover, following AMD3100 administration, Treg (both TIGIT $^{+}$and TIGIT $^{\text {neg }}$ ) numbers were significantly increased in the PB (Figure 5C). Importantly, in contrast to HSC mobilization, AMD3100 elicited higher numbers of Tregs in the PB and was superior to G-CSF in terms of Treg mobilization. Our earlier studies have demonstrated that G-CSF administration elicited not only increased Treg numbers in SCT grafts, but also an mRNA autophagy signature in these Tregs (19). We also noted that G-CSF induces autophagy in 
neutrophils, which is required for their effective mobilization following G-CSF administration (35). Thus, to examine whether G-CSF signaling induces autophagy in Tregs, we monitored LC3 accumulation in Tregs cultured with or without G-CSF and found that G-CSF exposure induced autophagy in both TIGIT $^{+}$and TIGIT ${ }^{\text {neg }}$ Tregs (Figure 5D). Taken together, the data demonstrate that G-CSF not only mobilizes Tregs into the periphery via the disruption of the CXCR4/SDF-1 axis, it additionally induces autophagy in Tregs, endowing these cells with an enhanced capacity for survival.

Autophagy-dependent Tregs are required to control GVHD. Since Tregs are critical for the establishment of tolerance after SCT, we analyzed the role of autophagy-dependent Tregs in protecting from GVHD after SCT with G-CSF-mobilized grafts (Figure 6A). Recipients receiving grafts from $\mathrm{Atg} \mathrm{7}^{\ell / f t}-\mathrm{FoxP}_{\mathrm{C}} \mathrm{cre} \mathrm{e}^{+}$mice developed accelerated GVHD mortality compared with mice receiving grafts from $W T$-FoxP $3 \mathrm{cre}^{+}$mice (Figure $6 \mathrm{~B}$ ) due to the development of severe GVHD within the GI tract (Figure 6C). Analysis of Treg populations 42 days after allogeneic SCT demonstrated a clear defect in Treg engraftment, which was most pronounced within the TIGIT $^{+}$Treg compartment in the BM in the recipients of Atg $^{7 f f}$-FoxP3cre ${ }^{+}$grafts (Figure 6D). We sought to confirm these finding in a second GVHD model, where identical numbers of Tregs could readily be transferred in the absence of G-CSF priming. Here we transplanted grafts comprising BM with or without supplementation with equal numbers of sort-purified splenic Tcon cells and Tregs from $W T-F o x P 3 c r e^{+}$or Atg $7^{f f l}$-FoxP3 $\mathrm{cre}^{+}$donors (Figure 7A). In this model, recipients of $A \operatorname{tg} 7^{f f t}$-FoxP3cre ${ }^{+} \mathrm{T}$ cell-replete grafts exhibited increased severity of GVHD from day 21 onward, with significantly reduced clinical scores and survival compared with recipients of $W T-F o x P 3 c r e+$ grafts (Figure 7, B and C). At day 28 after transplant, increased IFN- $\gamma$ levels were noted in the sera of animals transplanted with Atg $^{7 f f}-F o x P 3 c r e^{+}$grafts (Figure 7D). This was associated with an expansion of $\mathrm{CD}^{+}$Tcon cells in the BM (Figure 7E). Here pathology was due to excessive donor $\mathrm{T}$ cell alloreactivity, as recipients of $\mathrm{T}$ cell-depleted (TCD) WT-FoxP3cre ${ }^{+}$or Atg $7^{f f}$-FoxP3cre ${ }^{+}$grafts all survived without features of GVHD. Importantly, recapitulating our observations in the G-CSF SCT model, autophagy deficiency significantly impacted Treg reconstitution, which again was most striking in the BM and within the TIGIT ${ }^{+}$population. Within the spleen, only $\mathrm{TIGIT}^{+}$Tregs were significantly reduced, whereas both $\mathrm{TIGIT}^{+}$and $\mathrm{TIGIT}^{\text {neg }}$ Treg subsets were diminished in the BM. Thus, whereas in naive $A \operatorname{tg} 7-F o x P 3 c r e^{+}$mice the requirement for autophagy was predominantly associated with TIGIT $^{+}$Tregs, during GVHD both TIGIT $^{+}$and TIGIT $^{\text {neg }}$ Treg populations were impacted. This likely reflects plasticity in TIGIT expression by Tregs, as TIGIT ${ }^{\text {neg }}$ Tregs rapidly express TIGIT following adoptive transfer and in vitro activation $(30,36)$. As such, in an inflammatory environment, the Treg expression of TIGIT does not provide a reliable marker of autophagy-dependent Tregs.

\section{Discussion}

Tregs play a central role in the establishment of peripheral tolerance and immune homeostasis, and perturbation in Treg homeostasis is broadly associated with the development of systemic inflammation and autoimmunity. This is perhaps best exemplified in the setting of allogeneic SCT, where in both preclinical and clinical studies, the failure of Treg reconstitution or maintenance is implicated as causative mechanism in the development of severe GVHD. Although under intense investigation, the mechanisms controlling Treg development, homeostasis, stability, and survival remain unclear. The role for autophagy in the maintenance of immune homeostasis is increasingly recognized, and recently the intrinsic role of this pathway in Treg biology and for their antitumor response was reported by Wei et al. (23). This study demonstrated a specific requirement for autophagy within Tregs for lineage stability, via the inhibition of mTORC1 and c-Myc. Herein we confirm an intrinsic requirement of autophagy for the maintenance of Tregs in the periphery, and further identify a TIGIT ${ }^{+}$Treg subset that is uniquely dependent on autophagy at steady state. We also identify the BM as a reservoir for this autophagydependent TIGIT $^{+}$Treg subset, the ability of G-CSF to mobilize this subset from the BM, and their importance in the control of GVHD following SCT.

Using multiple models of autophagy deficiency, we confirmed that autophagy is dispensable for thymic Treg development and demonstrate that memory/activated Tregs exhibit an intrinsic and critical requirement for this process for their maintenance in the periphery. Thus, the lineage-specific ablation of autophagy in FoxP3-expressing cells significantly reduced Treg numbers in the periphery; however, the reduction was only partial and associated with a milder phenotype than observed in FoxP3-deficient (scurfy) mice or Treg-depleted mice. In Atg $^{f f f}-F_{0 x P} 3 \mathrm{cre}^{+}$mice, overt immunopathology was only evident in aged mice, although this was preceded by the activation and expansion of effector $\mathrm{T}$ cells. 
In contrast to the spleen, where Treg numbers were only reduced by $30 \%$, autophagy deficiency intrinsic to Tregs profoundly impacted the BM Treg compartment. Residual Tregs in the spleen of Atg $7^{f f l}$-FoxP $3 \mathrm{cre}^{+}$mice were hyperproliferative and expressed MCL-1, demonstrating active niche-filling behaviour (26), presumably evoked to repopulate the Treg compartment. Thus, autophagy is required not for Treg generation or proliferation, but rather for their survival. Further support of this is the fact that highly functional iTregs can be generated normally from autophagy-deficient Tcon cells.

Tregs display significant subset diversity in terms of developmental origin (thymic-derived natural Tregs versus iTregs), activation status (resting versus activated/memory), and rate of turnover (37). In contrast the compartmentalization of Treg subsets within specific organs remains a largely unexplored phenomenon. The expression of TIGIT on Tregs marks a highly activated subset with potent Th1/Th17 suppressive capacity $(30,31)$. Remarkably, TIGIT $^{+}$Tregs in both the spleen and BM expressed significantly lower levels of CD25, the IL-2 coreceptor. Since IL-2 plays a critical role in Treg maintenance (38), this finding further supports differential survival requirements in $\mathrm{TIGIT}^{+}$and $\mathrm{TIGIT}^{\text {neg }}$ Tregs. In this regard, we demonstrate that the survival of TIGIT $^{+}$Tregs is uniquely dependent on autophagy at steady state, and that these cells are highly enriched within the BM, explaining the preferential impact of autophagy deficiency on the BM Treg compartment. Furthermore, TIGIT ${ }^{+}$Tregs expressed lower levels of BCL-2 and higher LC3 protein and ATG7 transcripts compared with TIGIT ${ }^{\text {neg }}$ Tregs, supporting the notion that this Treg subset preferentially utilizes autophagy as a survival program.

Residency in the BM per se does not appear to require autophagy, as TIGIT $^{+}$Tregs from either the spleen or BM exhibited similar phenotype and basal levels of autophagic activity. It would thus appear that the stem cell niche represent an additional, hitherto unrecognized, niche for antigen-experienced Tregs whose survival is uniquely dependent on autophagy. In support of this concept, the BM forms a niche for long-lived HSCs and memory cells, such as plasmablasts and $\mathrm{CD} 4^{+}$and $\mathrm{CD} 8^{+}$memory $\mathrm{T}$ cells, each of which exhibits an intrinsic requirement for autophagy for their survival (39-42). We further demonstrate that TIGIT $^{+}$Tregs coexpress CXCR4, a chemokine receptor that facilitates migration to, and retention in, the BM, where stromal cells express high levels of SDF-1 (43), thus explaining the preferential enrichment of TIGIT $^{+}$Tregs at this site. G-CSF is known to disrupt CXCR4-SDF-1 interactions, facilitating the mobilization of cells (including HSCs and Tregs) anchored in the BM to the periphery (12). In naive mice, we show that therapeutic CXCR4 antagonists and G-CSF increase the number of Tregs in the periphery, resulting in enrichment of TIGIT $^{+}$Tregs, which in part explains the mRNA autophagy signature seen in splenic Tregs isolated from G-CSF-treated mice (19). This signature may also reflect the direct induction of autophagy in Tregs following G-CSF stimulation. In line with those observations, we have also recently demonstrated the requirement of autophagy for the mobilization of HSC by G-CSF (35). As G-CSF also invokes additional regulatory mechanisms, including tolerogenic dendritic cells, Th2 differentiation, and regulatory monocytes (44), regulation of autophagy within Tregs is likely one further beneficial effect in relation to tolerogenic properties. Indeed, autophagy deficiency in bone marrow-derived donor Tregs results in the failure of Treg homeostasis and exacerbates GVHD.

The identification of an autophagy-dependent TIGIT ${ }^{+}$Treg subset and the BM niche in which they reside represents an important advance for the transplant field, particularly given the fact that leukemia, the immunological target of SCT, also resides in this compartment. It is interesting to note that while Tregs are required for the induction of tolerance after SCT and stem cell engraftment (45), they also appear to be a target in GVHD (3), where tolerance has failed. BM failure is a characteristic feature of GVHD that is thought to be dependent on IFN- $\gamma$ signaling in the marrow stroma (46), and associated neutropenia may also result in high levels of G-CSF which may further disrupt the $\mathrm{TIGIT}^{+}$Tregs from this niche. Furthermore, the stem cell niche is known to be damaged in the context of GVHD (47), and the contribution of this to the failure of $\mathrm{TIGIT}^{+}$Treg homeostasis therein is an attractive concept to explain the development of cGVHD that now deserves further study. Manipulating the BM Treg population by ex vivo expansion and adoptive transfer seems challenging given the small numbers accessible. Alternatively, drugs that specifically enhance autophagy are likely to be very active in enhancing the survival of this population in vivo and deserve further study, and rapamycin is an obvious example. Unfortunately, it is difficult to fully dissect the ability of this agent to prevent GVHD by direct effects on other leukocyte populations such as Tcon cells or DCs mediated by mTOR inhibition from effects on Tregs via the induction of autophagy. Notably, autophagy is also required for the maintenance of $\mathrm{CD} 8^{+}$memory T cells (41), which are important effectors of both GVHD and GVL. Thus, approaches that globally induce autophagy after transplant must 
be approached with caution. Promisingly, however, a recent study has reported the beneficial effect of treatment with metformin, an autophagy-promoting drug, on survival in a model of GVHD (48). This effect was associated with inhibition of MTOR, a decrease in Th1/Th17 differentiation, and an increase in Tregs. Furthermore, the adoptive transfer of ex vivo expanded Tregs for the control of GVHD after SCT is an increasingly attractive cell therapy approach that has already shown efficacy in the clinic (49). Thus, an alternative strategy would be to invoke autophagy in the Treg product in vitro prior to adoptive transfer. In support of the feasibility of this approach, a recent preclinical study has demonstrated that priming Tregs with TNF in vitro enhanced their capacity to protect against GVHD (50).

Collectively, our results have identified a critical intrinsic requirement for autophagy in the maintenance of highly suppressive Helios ${ }^{+}$TIGIT $^{+}$Treg subset. The disruption of this pathway alters Tregs in the periphery, most strikingly in the BM, where $\mathrm{TIGIT}^{+}$Tregs are markedly enriched, resulting in the disruption of tolerance. These data identify autophagy as a potential therapeutic target to modulate Tregs for the promotion of tolerance after SCT and in other diseases characterized by a defect of the Treg population.

\section{Methods}

Supplemental Methods are available online with this article; doi:10.1172/JCI86850DS1.

Animals. C57BL/6 (H2D $\left.{ }^{\mathrm{b}}, \mathrm{CD} 45.2^{+}\right)$, B6.SJL-Ptprc ${ }^{a} \operatorname{eprc}^{b}\left(P T P r c^{\mathrm{a}}, \mathrm{H}-2 \mathrm{~b}, \mathrm{CD} 45.1^{+}\right), \mathrm{C} 57 \mathrm{BL} / 6-\mathrm{B} 6 . \mathrm{SJL}-\mathrm{Pt}-$ $\operatorname{prc}^{\mathrm{a}}\left(\mathrm{B} 6 \mathrm{x}\right.$ Ptprc $\left.{ }^{a}, \mathrm{H}-2 \mathrm{~b}, \mathrm{CD} 45.2^{+} \mathrm{CD} 45.1^{+}\right)$and B6D2F1 (H-2b/d,CD45.2) mice were purchased from Animal Resources Centre. B6.FoxP3cre (51), B6.IL-10-GFP, B6.FoxP3-RFP, HSC-SCL-Cre-ER ${ }^{T}$ (52), R26R-EY$F P$ (53), BALB/c.FoxP3-GFP mice were provided by Alexander Rudensky (Memorial Sloan Kettering Cancer Institute), and B6.FoxP3.LuciDTR4 (FoxP3-GFP) mice (54) were supplied by the QIMR Berghofer Animal Facility. B6.LC3-GFP (55) and B6.Atg5 $5^{\text {loxp/loxp }}$ (56) mice were provided by Noboru Mizushima (RIKEN BioResource Center). Atg $7^{f / f l}$ (57) mice were provided by Masaaki Komatsu (Tokyo Metropolitan Institute of Medical Science). FoxP3-Cre $e^{+}$mice were bred with $A \operatorname{tg} 7^{f / f l}$ to generate $A \operatorname{tg} 7^{f / f t}$-FoxP3cre $e^{+}$mice. Female mice were used between 8 and 13 weeks, unless otherwise indicated. All mice strains were backcrossed at least 10 times with C57BL/6 or BALB/c mice.

Antibodies and flow cytometry reagents. Phycoerythrin-conjugated (PE-conjugated) anti-CD44 (1M7), anti-ICOS (7E.17G9), anti-CD73 (TY/11-8), anti-CTLA4 (UC10-4B9), anti-FoxP3 (150D), anti-IL-10 (JES5-16E3), anti-CD90.2 (clone 53-2.1), Alexa Fluor 647-conjugated anti-FoxP3 (150D) and anti-BCL2 (BCl/10C4), FITC-conjugated anti-CD45.2 (clone 104), Alexa Fluor 700-conjugated anti-CD62L (MEL14), anti-CD4 (GK1.5) or anti-CD45.2 (clone 104), PE-Cy7-conjugated anti-CD3 (145-2C11), anti-CD8 (clone 53-6.7), anti-IFN- $\gamma$ (XMG1.2), PD-1 (RMPI-30), anti-CD45.1 (A20), brilliant violet (BV) 605conjugated streptavidin, anti-CD90.2 (clone 23-2.1), anti-IL-17 (TC11-18H10.1), Pacific blue-conjugated anti-Helios (22F6), anti-CD69 (H1.2F3) and allophycocyanin-conjugated (APC-conjugated) anti-mouse (RMG1-1), anti-GITR (YGITR765) and anti-CD3 (145-2C11), Alexa Fluor 647-conjugated anti-Mac-1 (M1/70), BV421-conjugated anti-Sca-1(D7), BV785-conjugated anti-B220 (RA3_6BD), BV650-conjugated anti-CD150 (TC15-12F12.3) Abs were purchased from BioLegend. Pecy7- or PerCP-Cy5.5- or Pacific blue-conjugated CD4 (RM4-5) or anti-CD8 (clone 53-6.7), Alexa Fluor 647-conjugated anti-Gr-1 (RB68C5; BD), brilliant ultraviolet (BUV) 395-conjugated anti-c-kit (2B8; BD), BV510-conjugated anti-CD48 (HM48-1; BD), PE-CF594-conjugated anti-CD3 (145-2C11), Alexa Fluor 700-conjugated anti-CD45.1 (A20), PE-conjugated anti-CD25 (7D4), anti-CD54 (3E2), anti-TIGIT (1G9), V500-conjugated anti-CD8 (clone 53-6.7), anti-CD45.2 (clone 104), PE-Cy7-conjugated anti-Ki67 (B56), and biotin-conjugated antiH2Dd (clone 34-2-12) Abs were purchased from BD Biosciences. Alexa Fluor 660-conjugated anti-TIGIT (GIGD7), anti-CXCR4 (2B11), PE- conjugated anti-TIGIT (GIGD7), APC-conjugated anti-KLRG1 (2F1), and eFluor 450-conjugated anti-FoxP3 (FJK-16s) Abs were purchased from eBioscience. Purified anti-LC3 (M152-3) Ab was purchased from MBL. Biotinylated anti-CD25 (7D4) Ab was purchased from BD Pharmingen. Anti-CD3 (2C11) and anti-CD28 (N3751) and MCL-1 Abs were produced in-house (58). 7-Aminoactinomycin D (7AAD) was purchased from Sigma-Aldrich. All intracellular cytokine and FoxP3 staining was performed using a FoxP3 Cytofix/Cytoperm Kit (eBioscience).

Flow cytometry acquisition was performed with a LSRFortessa cytometer (BD Biosciences), and data were analyzed using FlowJo software version 9.7.6. and version 10. Cell sorting was performed using a FACS ARIA III Cell Sorter (BD Biosciences) or using a MoFlo (DakoCytomation).

Measure of autophagic activity. Splenic $\mathrm{CD}^{+}$cells isolated from B6.FoxP3-GFP mice were purified by MACS bead (CD4) positive selection on columns according to the manufacturer's protocol (Miltenyi Bio- 
tec) and were cultured with or without CQ $(80 \mu \mathrm{M}$, Sigma-Aldrich) for 5 hours. Anti-CD4 surface staining was followed by intracellular staining with anti-LC3 Ab using the Cytofix/Cytoperm kit (BD Biosciences). The number and distribution of LC3 puncta was analyzed with the ImageStream ${ }^{\mathrm{X}}$ (Amnis). The images $(60 \times)$ and statistics were processed using IDEAS software (version 6.0, Amnis).

Cell preparation. For cytometry analysis of LC3 expression following G-CSF stimulation, splenic CD4 ${ }^{+}$ $\mathrm{T}$ cells from C57BL/6 mice were purified by MACS bead (CD4) positive selection according to the manufacturer's protocols (Miltenyi Biotec). Then $\mathrm{CD}^{+}$cells were incubated in vitro for 2 hours with or without G-CSF (Amgen) (100 ng/ml) with CQ (80 $\mu \mathrm{M}$, Sigma-Aldrich).

Histology. Organs were harvested, fixed in 10\% formalin ( 24 hours), preserved in $70 \%$ ethanol, embedded in paraffin, and sectioned $(5 \mu \mathrm{m})$. H\&E or Masson's trichrome staining was performed on sections as previously described (59). Stained sections were examined in a blinded fashion (by ADC) to determine the clinical score as previously published (60). Images were acquired using an Olympus BX51 microscope, Evolution MPVersion 5.0 camera, and QCapture software (QImaging).

Real-time quantitative PCR. Total RNA was extracted from sorted cells using the RNeasy Micro kit (QIAGEN), and reverse transcription was performed using SuperScript III Reverse Transcriptase (Life Technologies). RNA relative expression was determined using Taqman gene expression assays (taqman gene ex assays mto, mm00512209_m1 atg7; taqman gene ex assays mto, xs mm01545399_m1 hprt) (Life Technologies). The housekeeping gene HPRT was used to normalize the gene expression to the starting quantity of RNA. ATG7 mRNA expression was calculated using the $2^{-\Delta \Delta C t}$ method (61).

Mobilization methods. For initial mobilization experiments and analysis of LC3 levels (Figure 5, A and D), BALB/c.FoxP3-GFP mice received daily subcutaneous injections of recombinant human G-CSF (Amgen, $10 \mu \mathrm{g} / \mathrm{animal}$ ) or saline for 6 days. For comparison of the efficacy of G-CSF and AMD3100 Treg mobilization (Figure 5C), B6.FoxP3-GFP mice received a single subcutaneous injection of AMD3100 (Sigma-Aldrich, $30 \mu \mathrm{g} / 10 \mathrm{~g}$ body weight) 1 hour prior to harvest, or twice daily subcutaneous injections (6-8 hours apart) of recombinant human G-CSF $(500 \mu \mathrm{g} / \mathrm{kg} / \mathrm{d}$, for 4 consecutive days and harvested on day 5). Control mice received an equivalent volume of saline.

Stem cell transplantation. FLC and BMC mice were generated by transferring $1 \times 10^{6} \mathrm{WT}$ or Atg5 $5^{-/-}$fetal liver cells or Atg $7^{f / f} x H S C$-SCL-Cre-ER $R^{T} ; R 26 R$-EYFP BM into lethally irradiated (split dose 1,000 cGy) Ptprc ${ }^{a}$ recipients. FLC mice were examined 8 weeks after transplant. BMC mice were treated with tamoxifen chow (3 weeks) (400 mg/kg; Glen Forrest Stockfeeders), from 4 weeks after transplant, to induce Atg7 deletion and subsequent EYFP expression within HSCs (52). Mixed BMC mice were generated by injecting equal numbers of BM cells $\left(2 \times 10^{6}\right)$ from $W T-F o x P 3 c r e^{+}\left(\mathrm{CD} 45.2^{+}\right)$or Atg $^{7 / / f t}-F_{0 x P 3} \mathrm{cre}^{+}\left(\mathrm{CD} 45.2^{+}\right)$with CD $45.1^{+} \mathrm{CD} 45.2^{+}$(B6.SJL-Ptprc ${ }^{a} P e p c^{b}$ mice) into lethally irradiated $P t p r c^{a}\left(\mathrm{CD} 45.1^{+}\right.$) recipients.

For the SCT using G-CSF-mobilized graft, donor mice were treated with subcutaneous injections of recombinant human G-CSF (Amgen) (10 $\mu \mathrm{g}$ /animal) daily for 6 days. On day 0 , irradiated (1,100 cGy) B6D2F1 recipient mice and irradiated (1,000 cGy) Ptprc $^{a}$ recipients received approximately $10 \times 10^{6}$ G-CSF-mobilized splenic cells (analyzed by flow cytometry and cell count adjusted to inject $1.5 \times 10^{6}$ $\mathrm{CD}^{+}$cells/graft) from either G-CSF-treated $\mathrm{Atg}^{7 / / f-F o x P 3} \mathrm{cre}^{+}$or WT-FoxP3 $\mathrm{Cre}^{+}$mice. For the BMT, on day 0 , irradiated (1,100 cGy) B6D2F1 recipient mice received T cells purified using magnetic bead depletion (purity $>80 \%$ ) as previously described (62) consisting of sorted and combined $\mathrm{YFP}^{+}\left(0.75 \times 10^{5}\right)$ and YFPeg $^{\text {neg }}\left(0.5 \times 10^{6}\right)$ T cells from either Atg $7^{7 / f t}-F_{0 x P} 3 c r e^{+}$or $W T$-FoxP3cre ${ }^{+}$mice. Recipient mice received 5 $\times 10^{6} \mathrm{~T}$ cell-depleted BM from Atg $7^{f / f}-F_{0} x P 3 c r e^{+}$or $W T-F o x P 3 c r e^{+}$mice. T cell depletion of BM cells was performed as previously described (62). Mice were monitored daily to evaluate clinical GVHD scores as previously published (63). Briefly, weight loss, posture, activity, fur texture, and skin integrity were measured. Mice with a score greater than or equal to 6 were culled, and the date of the following day is recorded as the death date.

$I F N-\gamma$ quantification. IFN- $\gamma$ quantification in sera was performed using the BD Cytometric Bead Array system (BD Biosciences Pharmingen), according to the manufacturer's protocols.

\section{Statistics}

Data are shown as mean \pm SEM. Statistical significance was determined using an unpaired 2-tailed Mann-Whitney $U$ test, Wilcoxon matched-paired test, log-rank (Mantel-Cox) test, or paired $t$ test when appropriate $\left({ }^{*} P<0.05 ;{ }^{* *} P<0.01 ;{ }^{* * *} P<0.001,{ }^{* * * *} P<0.0001\right)$. Statistical analyses were performed using GraphPad Prism software version 6.01. 
Study approval. All experiments were performed under approval of the QIMR Animal Ethics Committee (ethics number P2096, P832).

\section{Author contributions}

KPAM, LLT, LLE, BC, SKN, CMH, GRH, BRB, and DG conceived and designed the experiments; LLT, KEL, LLE, JN, BC, CMH, SKN, KAA, BT, BCN, MM, RDK, ML, SJB, FSFG, CT, and KPAM performed the experiments; LLT, KPAM, LLE, CMH, ADC, and SJB analyzed the data; KPAM, GRH, ADC, SWL, MT, DG, and CRE contributed reagents/materials; and LLT, KPAM, BRB, and GRH wrote the manuscript.

\section{Acknowledgments}

This work is supported by NHMRC project grant A1089138.

Address correspondence to: Kelli P.A. MacDonald, QIMR Berghofer Medical Research Institute, 300 Herston Road, Brisbane, Queensland 4006, Australia. Phone: 61.0.7.3362.0404; E-mail: Kelli.MacDonald@qimrberghofer.edu.au.

1. Beres AJ, Drobyski WR. The role of regulatory T cells in the biology of graft versus host disease. Front Immunol. 2013;4:163

2. Zorn E, et al. Reduced frequency of FOXP3 + CD4+CD25+ regulatory T cells in patients with chronic graft-versus-host disease. Blood. 2005;106(8):2903-2911.

3. Matsuoka K, et al. Low-dose interleukin-2 therapy restores regulatory T cell homeostasis in patients with chronic graft-versushost disease. Sci Transl Med. 2013;5(179):179ra43.

4. Koreth J, et al. Efficacy, durability, and response predictors of low-dose interleukin-2 therapy for chronic graft-versus-host disease. Blood. 2016;128(1):130-137.

5. Zhao D, et al. In vivo-activated CD103+CD4+ regulatory T cells ameliorate ongoing chronic graft-versus-host disease. Blood. 2008;112(5):2129-2138.

6. Taylor PA, Lees CJ, Blazar BR. The infusion of ex vivo activated and expanded CD4(+)CD25(+) immune regulatory cells inhibits graft-versus-host disease lethality. Blood. 2002;99(10):3493-3499.

7. Hoffmann P, Ermann J, Edinger M, Fathman CG, Strober S. Donor-type CD4(+)CD25(+) regulatory T cells suppress lethal acute graft-versus-host disease after allogeneic bone marrow transplantation. J Exp Med. 2002;196(3):389-399.

8. Giorgini A, Noble A. Blockade of chronic graft-versus-host disease by alloantigen-induced CD4+CD25+Foxp3+ regulatory T cells in nonlymphopenic hosts. J Leukoc Biol. 2007;82(5):1053-1061.

9. McDonald-Hyman C, Flynn R, Panoskaltsis-Mortari A, et al. Therapeutic regulatory T-cell adoptive transfer ameliorates established murine chronic GVHD in a CXCR5 dependent manner [published online ahead of print July 6, 2016]. Blood. doi: 10. 1182/blood-2016-05-715896.

10. Leveque-El Mouttie L, et al. Corruption of dendritic cell antigen presentation during acute GVHD leads to regulatory T-cell failure and chronic GVHD. Blood. 2016;128(6):794-804.

11. Hill GR, et al. Stem cell mobilization with G-CSF induces type 17 differentiation and promotes scleroderma. Blood. 2010;116(5):819-828.

12. Petit I, et al. G-CSF induces stem cell mobilization by decreasing bone marrow SDF-1 and up-regulating CXCR4. Nat Immunol. 2002;3(7):687-694.

13. Peled A, et al. Dependence of human stem cell engraftment and repopulation of NOD/SCID mice on CXCR4. Science. 1999;283(5403):845-848.

14. Cashman J, Clark-Lewis I, Eaves A, Eaves C. Stromal-derived factor 1 inhibits the cycling of very primitive human hematopoietic cells in vitro and in NOD/SCID mice. Blood. 2002;99(3):792-799.

15. Morris ES, MacDonald KP, Hill GR. Stem cell mobilization with G-CSF analogs: a rational approach to separate GVHD and GVL? Blood. 2006;107(9):3430-3435.

16. Stem Cell Trialists' Collaborative Group. Allogeneic peripheral blood stem-cell compared with bone marrow transplantation in the management of hematologic malignancies: an individual patient data meta-analysis of nine randomized trials. J Clin Oncol. 2005;23(22):5074-5087.

17. Anasetti C, et al. Peripheral-blood stem cells versus bone marrow from unrelated donors. N Engl J Med. 2012;367(16):1487-1496.

18. Serody JS, Hill GR. The IL-17 differentiation pathway and its role in transplant outcome. Biol Blood Marrow Transplant. 2012;18(1 Suppl):S56-S61.

19. MacDonald KP, et al. Modification of $\mathrm{T}$ cell responses by stem cell mobilization requires direct signaling of the $\mathrm{T}$ cell by G-CSF and IL-10. J Immunol. 2014;192(7):3180-3189.

20. Mizushima N. Autophagy: process and function. Genes Dev. 2007;21(22):2861-2873.

21. Abdelaziz DH, Khalil H, Cormet-Boyaka E, Amer AO. The cooperation between the autophagy machinery and the inflammasome to implement an appropriate innate immune response: do they regulate each other? Immunol Rev. 2015;265(1):194-204

22. Bronietzki AW, Schuster M, Schmitz I. Autophagy in T-cell development, activation and differentiation. Immunol Cell Biol. 2015;93(1):25-34

23. Wei J, et al. Autophagy enforces functional integrity of regulatory $\mathrm{T}$ cells by coupling environmental cues and metabolic homeostasis. Nat Immunol. 2016;17(3):277-285 
24. Klionsky DJ et al. Guidelines for the use and interpretation of assays for monitoring autophagy. Autophagy. 2012;8(4):445-544.

25. Zhao Z, et al. Autophagosome-independent essential function for the autophagy protein Atg5 in cellular immunity to intracellular pathogens. Cell Host Microbe. 2008;4(5):458-469.

26. Pierson W, et al. Antiapoptotic Mcl-1 is critical for the survival and niche-filling capacity of Foxp3(+) regulatory T cells. Nat Immunol. 2013;14(9):959-965.

27. Franckaert D, et al. Promiscuous Foxp3-cre activity reveals a differential requirement for CD28 in Foxp3(+) and Foxp3(-) T cells. Immunol Cell Biol. 2015;93(4):417-423.

28. Thornton AM, et al. Expression of Helios, an Ikaros transcription factor family member, differentiates thymic-derived from peripherally induced Foxp3+ T regulatory cells. J Immunol. 2010;184(7):3433-3441.

29. Käser T, Mair KH, Hammer SE, Gerner W, Saalmüller A. Natural and inducible Tregs in swine: Helios expression and functional properties. Dev Comp Immunol. 2015;49(2):323-331.

30. Joller N, et al. Treg cells expressing the coinhibitory molecule TIGIT selectively inhibit proinflammatory Th1 and Th17 cell responses. Immunity. 2014;40(4):569-581.

31. Joller N, et al. Cutting edge: TIGIT has T cell-intrinsic inhibitory functions. J Immunol. 2011;186(3):1338-1342.

32. Re F, Srinivasan R, Igarashi T, Marincola F, Childs R. Green fluorescent protein expression in dendritic cells enhances their immunogenicity and elicits specific cytotoxic T-cell responses in humans. Exp Hematol. 2004;32(2):210-217.

33. Zou L, et al. Bone marrow is a reservoir for CD4+CD25+ regulatory T cells that traffic through CXCL12/CXCR4 signals. Can cer Res. 2004;64(22):8451-8455.

34. Ukena SN, et al. Human regulatory T cells of G-CSF mobilized allogeneic stem cell donors qualify for clinical application. PLoS One. 2012;7(12):e51644.

35. Leveque-E1 Mouttie L, et al. Autophagy is required for stem cell mobilization by G-CSF. Blood. 2015;125(19):2933-2936.

36. Yu X, et al. The surface protein TIGIT suppresses $\mathrm{T}$ cell activation by promoting the generation of mature immunoregulatory dendritic cells. Nat Immunol. 2009;10(1):48-57.

37. Luo CT, Liao W, Dadi S, Toure A, Li MO. Graded Foxo1 activity in Treg cells differentiates tumour immunity from spontaneous autoimmunity. Nature. 2016;529(7587):532-536.

38. Boyman O, Sprent J. The role of interleukin-2 during homeostasis and activation of the immune system. Nat Rev Immunol. 2012;12(3):180-190.

39. Warr MR, et al. FOXO3A directs a protective autophagy program in haematopoietic stem cells. Nature. 2013;494(7437):323-327.

40. Arnold J, Murera D, Arbogast F, Fauny JD, Muller S, Gros F. Autophagy is dispensable for B-cell development but essential for humoral autoimmune responses. Cell Death Differ. 2016;23(5):853-864.

41. Chen M, et al. Essential role for autophagy in the maintenance of immunological memory against influenza infection. Nat Med. 2014;20(5):503-510

42. Xu X, et al. Autophagy is essential for effector CD8(+) T cell survival and memory formation. Nat Immunol. 2014;15(12):11521161.

43. Ponomaryov T, et al. Induction of the chemokine stromal-derived factor-1 following DNA damage improves human stem cell function. J Clin Invest. 2000;106(11):1331-1339.

44. Yang JZ, Zhang JQ, Sun LX. Mechanisms for T cell tolerance induced with granulocyte colony-stimulating factor. Mol Immunol. 2016;70:56-62.

45. Fujisaki J, et al. In vivo imaging of Treg cells providing immune privilege to the haematopoietic stem-cell niche. Nature. 2011;474(7350):216-219.

46. Delisle JS, Gaboury L, Bélanger MP, Tassé E, Yagita H, Perreault C. Graft-versus-host disease causes failure of donor hematopoiesis and lymphopoiesis in interferon-gamma receptor-deficient hosts. Blood. 2008;112(5):2111-2119.

47. Shono Y, et al. Bone marrow graft-versus-host disease: evaluation of its clinical impact on disrupted hematopoiesis after allogeneic hematopoietic stem cell transplantation. Biol Blood Marrow Transplant. 2014;20(4):495-500.

48. Park MJ, et al. Metformin attenuates graft-versus-host disease via restricting mammalian target of rapamycin/signal transducer and activator of transcription 3 and promoting adenosine monophosphate-activated protein kinase-autophagy for the balance between T helper 17 and Tregs. Transl Res. 2016;173:115-130.

49. Brunstein CG, et al. Infusion of ex vivo expanded T regulatory cells in adults transplanted with umbilical cord blood: safety profile and detection kinetics. Blood. 2011;117(3):1061-1070.

50. Pierini A, et al. TNF- $\alpha$ priming enhances CD4+FoxP3+ regulatory T-cell suppressive function in murine GVHD prevention and treatment. Blood. 2016;128(6):866-871.

51. Rubtsov YP, et al. Regulatory T cell-derived interleukin-10 limits inflammation at environmental interfaces. Immunity. 2008;28(4):546-558.

52. Göthert JR, et al. In vivo fate-tracing studies using the Scl stem cell enhancer: embryonic hematopoietic stem cells significantly contribute to adult hematopoiesis. Blood. 2005;105(7):2724-2732.

53. Srinivas S, et al. Cre reporter strains produced by targeted insertion of EYFP and ECFP into the ROSA26 locus. BMC Dev Biol. $2001 ; 1: 4$.

54. Suffner J, et al. Dendritic cells support homeostatic expansion of Foxp3+ regulatory T cells in Foxp3.LuciDTR mice. J Immunol. 2010;184(4):1810-1820.

55. Mizushima N, Yamamoto A, Matsui M, Yoshimori T, Ohsumi Y. In vivo analysis of autophagy in response to nutrient starvation using transgenic mice expressing a fluorescent autophagosome marker. Mol Biol Cell. 2004;15(3):1101-1111.

56. Kuma A, et al. The role of autophagy during the early neonatal starvation period. Nature. 2004;432(7020):1032-1036.

57. Komatsu M, et al. Impairment of starvation-induced and constitutive autophagy in Atg7-deficient mice. J Cell Biol. 2005;169(3):425-434.

58. Zhang $\mathrm{P}$, et al. Induced regulatory $\mathrm{T}$ cells promote tolerance when stabilized by rapamycin and IL-2 in vivo. J Immunol. 2013;191(10):5291-5303.

59. Alexander KA, et al. CSF-1-dependant donor-derived macrophages mediate chronic graft-versus-host disease. J Clin Invest. 
2014;124(10):4266-4280.

60. MacDonald KP, et al. Donor pretreatment with progenipoietin-1 is superior to granulocyte colony-stimulating factor in preventing graft-versus-host disease after allogeneic stem cell transplantation. Blood. 2003;101(5):2033-2042.

61. Livak KJ, Schmittgen TD. Analysis of relative gene expression data using real-time quantitative PCR and the 2(-Delta Delta C(T)) Method. Methods. 2001;25(4):402-408.

62. Burman AC, et al. IFNgamma differentially controls the development of idiopathic pneumonia syndrome and GVHD of the gastrointestinal tract. Blood. 2007;110(3):1064-1072.

63. Hill GR, Crawford JM, Cooke KR, Brinson YS, Pan L, Ferrara JL. Total body irradiation and acute graft-versus-host disease: the role of gastrointestinal damage and inflammatory cytokines. Blood. 1997;90(8):3204-3213. 